\title{
Dp-brane Dynamics and Thermalization in Type IIB Ben Ami-Kuperstein-Sonnenschein Models
}

\author{
Dariush Kaviani \\ School of Particles and Accelerators, Institute for Research in Fundamental Sciences (IPM), P.O. Box 19395-5531, Tehran, Iran \\ Correspondence should be addressed to Dariush Kaviani; dariush@ipm.ir
}

Received 3 March 2020; Accepted 5 May 2020; Published 11 June 2020

Academic Editor: Elias C. Vagenas

Copyright (C) 2020 Dariush Kaviani. This is an open access article distributed under the Creative Commons Attribution License, which permits unrestricted use, distribution, and reproduction in any medium, provided the original work is properly cited. The publication of this article was funded by $\mathrm{SCOAP}^{3}$.

We extend and complete our analysis arXiv:1608.02380 and study the induced world volume metrics and Hawking temperatures of all type IIB rotating probe Dp-branes, dual to the temperature of different flavors at finite R-charge, in the Ben Ami-KupersteinSonnenschein holographic models including the effects of spontaneous conformal and chiral flavor symmetry breakdown. The model embeds type IIB probe flavor Dp-branes into the Klebanov-Witten gravity dual of conformal gauge theory, with the embedding parameter, given by the minimal radial extension of the probes, dual to the IR scale of conformal and chiral flavor symmetry breakdown. We show that when the minimal extension is positive definite, the induced world volume metrics of type IIB rotating probe branes admit thermal horizons and Hawking temperatures despite the absence of black holes in the bulk subject to the world volume and topology of the nontrivial internal cycle wrapped by the probe. We also derive the energystress tensor of the thermal probes and study their backreaction and energy dissipation. We show that at the IR scale the backreaction is nonnegligible and find the energy can flow from the probes to the bulk, dual to the energy dissipation from the flavor sectors into the gauge theory.

\section{Introduction}

Nonequilibrium steady states are of great interest in many branches of physics, such as heavy-ion physics (thermalization of the quark-gluon plasma), condensed matter physics (quenches of cold atom systems), and cosmology (nonequilibrium phase transitions and the Kibble-Zurek mechanism). Studying such states by standard field theory techniques is prohibitively difficult (except for special cases involving conformal symmetry or integrability); however, they can be easily constructed in string theory by using gauge/gravity duality with D-brane dynamics. Nonetheless, nonequilibrium states have been mainly constructed in gravity duals of conformal gauge theories and more general and realistic examples of such states including the effects of spontaneous conformal and chiral symmetry breakdown remain scarce.

In gauge/gravity duality, ref. [1-3] (see also ref. $[4,5]$ ), the pure gravity dual of $\mathcal{N}=4$ conformal gauge theory is produced by taking the near horizon limit of $N$ backreacting
D3-branes at a smooth point of the transverse space described by the $a d S_{5} \times S^{5}$ background metric. The extension of this solution to holographic QCD-like solutions includes the addition of flavored quarks to the pure gauge theory, by embedding additional $N_{f}$ flavor branes into the pure gravity dual, taking the probe limit, $N_{f} \ll N$, ref. [6] (see also ref. $[4,5])$. Such holographic setup including probes, ref. $[7,8]$, has been used to model flavor physics, ref. [6, 9-16], and quantum critical phenomena, ref. [17-24], complementary to other works on charged adS black holes, ref. [25-30]. Such phenomena have also been studied at zero temperature in refs. [31-33]. In order to construct nonequilibrium systems in such flavored holographic models, one then notes that when the flavored quark sector (dual to the probe flavor branes) admits finite temperature, while the pure gauge theory itself (dual to the pure gravity solution) is at zero temperature, the system describes nonequilibrium steady states. The prime examples of such nonequilibrium steady states have been constructed in ref. [34] (see also refs. 
[35-53]), by embedding type IIB probe flavor Dp-branes $(p=1,3,5,7)$ into the $a d S_{5} \times S^{5}$ gravity dual of conformal gauge theory. (For $p=7$, the probe fills all the dual spacetime directions and the gauge theory is given by conformal field theory (CFT). For $p<7$, the probes are localized in some dual spacetime directions and the gauge theory is given by defect conformal field theory (dCFT)). In ref. [34] (see also refs. [35-53]) rotating probe $D p$-brane solutions have been constructed and shown that when spin is turned on, the induced world volume metrics on all type IIB rotating probe Dpbranes in $a d S_{5} \times S^{5}$ admit thermal horizons and Hawking temperatures despite the absence of black holes in the bulk. It was then noted in ref. [34] that by taking into account the backreaction of the rotating Dp-branes solutions to the gravity dual of (d)CFT, one naturally expects all such type IIB branes to form mini black holes in the bulk, in the probe limit. By gauge/gravity duality, this means that when the flavor sector of the gauge theory gets R-charged, it becomes thermal and thereby the gauge theory couples to thermal objects and describes locally thermal vacua. Since the gauge theory itself is at zero temperature, while its flavor sector, given, for instance, by a magnetic monopole $(p=1)$ or a quark $(p=7)$, is at finite temperature, it was concluded in ref. [34] such systems describe nonequilibrium steady states. However, by computing the energy-stress tensor, it has been shown in ref. [34] that the energy of the probe (defect) flavor brane will eventually dissipate into the bulk and form, with the large backreaction in the IR, a (localized) black hole in the bulk. By gauge/gravity duality, this means that the energy from the (defect) flavor sector will eventually dissipate into the conformal gauge theory ( $\mathcal{N}=4 \mathrm{SYM})$.

This analysis has been extended in refs. [54, 55], by studying the induced world volume metrics on rotating probe D-branes embedded in more general and realistic gauge/gravity dualities, including warped Calabi-Yau throats, refs. [56-58] (see also refs. [59-63]). The warped throat geometry is produced by placing $N$ regular D3-branes and $M$ D5-branes (fractional D3-branes) at the generic CalabiYau singularity-the conifold tip point, [64], and describes the gravity dual of $S U(N+M) \times S U(N)$ gauge theory which breaks some supersymmetry and conformal invariance. The $\mathcal{N}=1$ duality includes confinement and chiral symmetry breakdown in the regular IR, where it describes the Klebanov-Strassler (KS) solution, [56], and admits RG cascade in the singular UV, where it describes the KlebanovTseytlin (KT) solution, [57]. The duality is conformal in the singular UV without fractional D3-branes, where it describes the Klebanov-Witten (KW) solution, [58]. In refs. [54, 55] it has been shown that when spin is turned on, the induced worldvolume metrics on rotating probe Dp-branes $(p=1,7$ ) in gravity duals of gauge theories with conformal and chiral symmetry breakdown, refs. [56-58], also admit thermal horizons and Hawking temperatures despite the absence of black holes in the throat. However, it has been shown there that the scale and behavior of the horizon and temperature change dramatically with the scale of conformal and chiral symmetry breakdown, implying that horizons and temperatures of expected features form in the singular UV and not in the regular confining IR. It has been then noted in refs. $[54,55]$ that by taking into account the backreaction of the rotating brane solutions to the throat background, one expects such solu- tions to form mini black holes in the bulk, in the probe limit. By gauge/gravity duality, this means that when the flavor sector of the gauge theory gets R-charged, it becomes thermal and thereby the gauge theory couples to thermal objects. Since the gauge theory itself is at zero temperature, while its flavor sector is at finite temperature, it was concluded in refs. $[54,55]$ that such systems describe nonequilibrium steady states in the gauge theory conformal and chiral symmetry breakdown. However, by computing the energy-stress tensor of the rotating branes, it has been found there that the energy of the branes will eventually dissipate into the bulk and form, with the large backreaction at small radii, a black hole in the throat. By gauge/gravity duality, it was then concluded in refs. $[54,55]$ that the energy from the flavor sector will eventually dissipate into the gauge theory conformal and chiral symmetry breakdown $(\mathscr{N}=1 \mathrm{SYM})$.

The aim of this work is to generalize and complete such previous analysis by studying thermalization in new embeddings dual to new gauge field theories. Our particular aim is to complete the analysis of ref. [55] by studying in its configuration thermalization for new embeddings involving the rest of probe branes in the theory. Thus by duality, our aim is to find novel examples and features of thermalization in new field theories with spontaneous conformal and chiral flavor symmetry breakdown.

The holographic model we consider is the Ben-AmiKuperstein-Sonnenschein (BKS) embedding model of D3and D5-branes, [65] (see also ref. [66, 67]), dual to dCFTs. The model follows the earlier Kuperstein-Sonnenschein holographic embedding model of D7-branes, ref. [68] (see also ref. $[69,70]$ and ref. [71, 72]), dual to CFT, which was motivated by the Sakai-Sugimoto model of D8-branes, ref. $[73,74]$. These models, ref. $[65,68]$, embed the $N_{f}$ probe Dp-branes $(p=3,5,7)$ and $N_{f}$ probe anti-Dp-branes into the KW gravity dual, ref. [58], of $\mathcal{N}=1$ gauge theory (dCFTS and CFT) including fundamental quarks and antiquarks (see also refs. [75-77] — considering holomorphic embeddings of D7-branes into the KS solution dual to supersymmetric gauge theory without flavor chiral symmetry breaking, and refs. [78-83] -studying the backreaction of D7-branes in the KS \& KW solutions). The quarks and antiquarks transform, respectively, in the fundamental representation of $U\left(N_{f}\right)_{L}$ and $U\left(N_{f}\right)_{R}$, resulting in a non-Abelian $U\left(N_{f}\right)_{L} \times$ $U\left(N_{f}\right)_{R}$ gauge symmetry on the gravity side, corresponding to a global chiral symmetry on the field theory side. This chiral symmetry, together with the conformal flavor symmetry, get spontaneously broken, when on the gravity side the Dp-branes and anti-Dp-branes extend from the UV and merge smoothly into a single curved $D p$-brane in the IR where only one $U\left(N_{f}\right)_{D}$ factor survives. The embedding then describes a probe $D p$-brane of $\mathrm{U}$-shape where the probe extends from the infinite far UV boundary to the minimal extension in the IR from where bends back to the boundary. The minimal extension measures the minimal separation between the probe and the background branes in the nearhorizon limit of at the conifold point and is dual to the IR 
scale conformal and chiral flavor symmetry breakdown which sets the VEV deformations in the dual gauge theory. In the U-shape configuration, the induced metric on the probe has no adS factor, and the embedding is dual to gauge theory conformal and chiral flavor symmetry breakdown. When the minimal extension shrinks to zero, the embedding forms a V-shape as it appears as a disconnected Dp-brane/ anti- $D p$-brane pair, the induced metric on the probe is adS -like and the embedding is dual to gauge theory conformal and chiral symmetry restoration. The probe also describes a SUSY breaking $D p$-brane/anti- $D p$-brane pair, merged in the bulk at minimal extension, and guarantees tadpole cancellation on the transverse space because the $D p$-brane and anti$D p$-brane differ only in orientation and the total $D p$ charge annihilates on the transverse sphere. As discussed in $\$ 2$, the BKS setup cannot be realized in the well-used $a d S_{5} \times S^{5}$ background, but instead it can be accomplished in the KW background, $a d S_{5} \times T^{1,1}$. Furthermore, we modify the BKS models by turning on additional spin on the probes dual to exciting R-charge in the flavor sectors of the gauge theory.

The motivation of this work is the fact that in such new embeddings the induced worldvolume metrics on rotating probe D3- and D5-branes in KW, if describing black hole geometries, are then expected to give the Hawking temperatures dual to the temperatures of flavors now in dCFTs (unlike D7 in KW which is a CFT) with spontaneous conformal and chiral flavor symmetry breakdown. Since the gauge field theory itself is at zero temperature while its defect flavor sector is at finite temperature, such systems exemplify novel nonequilibrium steady states in dCFTs with conformal and chiral flavor symmetry breakdown. The motivation is thus to construct and classify all such novel examples of nonequilibrium systems in KW.

The main results we find are as follows. We find that when the embedding is U-like and additional spin is turned on, the induced world volume metrics on rotating probes describe the black hole geometry subject to the worldvolume and topology of the nontrivial internal cycle wrapped by the probe. By gauge/gravity duality, we then find that when the conformal and chiral flavor symmetry gets broken and the flavor sector gets in addition R-charged, it becomes thermal subject to the type of flavor and codimension of dual spacetime where the flavors fields reside. We thus find that such nonequilibrium states are classified according to the kind of field theory (CFT or dCFT) and type of flavor inserted into the theory. Nonetheless, by computing the energy-stress tensor of the U-like embedded thermal probes, we find there that the energy of the brane will eventually dissipate into the bulk and form, with the large backreaction in the IR, a black hole in the KW throat. By gauge/gravity duality, we thus find that the energy from the flavor sector will eventually dissipate into the gauge theory conformal and chiral flavor symmetry breakdown.

The paper is organized as follows. In Sec. 2, we review the type IIB BKS models. In Sec. 3, we modify the BKS models and compute the induced world volume metrics and Hawking temperatures of rotating probes. We also compute the energy-stress tensors of the thermal probes and analyse their backreaction and energy dissipation. In Sec. 4, we summarize and discuss our results with future outlook.

\section{Review of the BKS Models}

In this section, we first review the relevant aspects of the KW gravity dual of conformal gauge theory, ref. [58]. We then review the Kuperstein-Sonnenschein and BKS embeddings of U-like probe flavor branes in the KW gravity dual of CFT and dCFTs with spontaneous conformal and chiral flavor symmetry breakdown ref. $[65,68]$.

2.1. The $K W$ Solution. The particular ten-dimensional holographic string solution we are interested in is the KW solution, [58], resulting from taking the near horizon limit of a stack of $\mathrm{N}$ background D3-branes on the conifold point. The conifold is a singular and noncompact Calabi-Yau threefold described, in terms of complex coordinates $z_{n} \in \mathbb{C}^{4}$, by the constraint and three-form as:

$$
\sum_{n=1}^{4} z_{n}^{2}=0, \Omega=\frac{d z_{2} \wedge d z_{3} \wedge d z_{4}}{z_{1}}
$$

The constraint equation in (1) describes a singular noncompact cone with symmetry $S O(4) \times U(1) \simeq S U(2) \times$ $S U(2) \times U(1)$, and the three-form in (1) is charged under the $U(1)$ R-symmetry. The base of the cone is a fivedimensional Einstein manifold, $X_{5}=T^{1,1}$, of topology $S O(4) /$ $U(1) \simeq S U(2) \times S U(2) / U(1)$, which can be identified by intersecting the constraint in (1) with the unit three-sphere $\sum_{n=1}^{4}\left|z_{n}\right|^{2}=1$.

The constraint equation in (1) can recast its form as:

$$
\operatorname{det}_{i, j} z_{i j}=0 \text {, with } z_{i j}=\sum_{n} \sigma_{i j}^{n} z_{n} .
$$

Here, $\sigma^{n}$ denotes the Pauli matrices with $n=1,2,3$ and $\sigma^{4}=i$ I. To solve Eq. (2), one may use unconstrained variables $z_{i j}=a_{i} b_{j}$, where $a_{i}, b_{j}$ denotes complex scalars with $i, j=1,2$. These scalars poses an additional $S U(2) \times S U(2)$ global symmetry, which is quotiented by the $U(1)$ symmetry via $a_{i} \longrightarrow \exp (i \alpha) a_{i}$ and $b_{j} \longrightarrow \exp (-i \alpha) b_{j}$, so that the global symmetry is $S U(2) \times S U(2) / U(1)$.

To construct the gauge field theory of the solution, one may use $a_{i}, b_{j}$ and note the following points. First, since the conifold is a Calabi-Yau manifold, it preserves by the virtue of Killing spinor equations, one-quarter of the original supersymmetry. Thus, the solution has $\mathcal{N}=1$ supersymmetry and one may associate chiral $\mathcal{N}=1$ superfields $A_{i}, B_{j}$ with $i, j=1,2$ to the complex scalars $a_{i}, b_{j}$. Second, further to the $S U(2) \times S U(2) / U(1)$ global symmetry discussed above, the solution has a $U(1)_{R}$ symmetry. Third, the field theory exemplifies a quiver gauge theory. Namely, the field theory of $N$ D3-branes placed on the conifold tip point has a $S U(N) \times S U(N)$ gauge symmetry where $A_{i}$ and $B_{j}$ transform in the $(\mathbf{N}, \overline{\mathbf{N}})$ and $(\overline{\mathbf{N}}, \mathbf{N})$ representation, respectively. Fourth, anomaly cancellation in $U(1)_{R}$ requires $A_{i}$ and $B_{j}$ 
have $\mathrm{R}$-charge $1 / 2$, respectively. Thus, the gauge theory also includes a marginal superpotential $W=\varepsilon^{i j} \varepsilon^{k l} \operatorname{Tr} A_{i} B_{k} A_{j} B_{l}$ which is uniquely fixed by the symmetries modulo an overall factor.

To construct the gravity dual of this gauge theory, one notes that Eq. (2) describes a cone over the base of coset space $T^{1,1}=S U(2) \times S U(2) / U(1)$. One also notes that the $z_{i j}$ can be explicitly parameterized by five Euler angels $\left(\psi, \phi_{i}, \theta_{i}\right)$ with $i=1,2$ (not written out here) whereby the metric on the conifold then takes the form [64]:

$$
d s_{T^{1,1}}^{2}=\frac{1}{9}\left(d \psi+\sum_{i=1}^{2} \cos \theta_{i} d \phi_{i}\right)^{2}+\frac{1}{6} \sum_{i=1}^{2}\left(d \theta_{i}^{2}+\sin ^{2} \theta_{i} d \phi_{i}^{2}\right)
$$

Here, $0 \leq \psi \leq 4 \pi, 0 \leq \phi_{i} \leq 2 \pi$ and $0 \leq \theta_{i} \leq \pi$. It is clear from (3) that the $T^{1,1}$ base is a $S^{1}$ bundle over $S^{2} \times S^{2}$. It is also clear from (3) that the $T^{1,1}$ base has the topology $S^{2} \times S^{3}$ where the three-cycle $S^{3}$ can be parameterized by $\theta_{1}=\phi_{1}=$ 0 and the two-cycle $S^{2}$ by $\psi=0, \theta_{1}=\theta_{2}, \phi_{1}=-\phi_{2}$, with both $S^{2}$ and $S^{3}$ shrinking to zero size at the tip of the cone.

The alternative way of writing the metric on the conifold is [84]:

$$
\begin{aligned}
d s_{T^{1,1}}^{2}= & \frac{r^{2}}{3}\left[\frac{1}{4}\left(\Omega_{1}^{2}+\Omega_{2}^{2}\right)+\frac{1}{3} \Omega_{3}^{2}+\left(d \theta-\frac{1}{2} \Omega_{2}\right)^{2}\right. \\
& \left.+\left(\sin \theta d \phi-\frac{1}{2} \Omega_{1}\right)^{2}\right] .
\end{aligned}
$$

Here, $r$ is the radial coordinate of the conifold (1), $\theta$ and $\phi$ parameterize the $S^{2}$, and $\Omega_{i}$ are one-forms parameterizing the $S^{3}$. The $\Omega_{i}$ s can be represented by Maurer-Cartan oneforms $w_{i}$ via two $S O(3)$ matrices (not written out here) parameterized by $\theta$ and $\phi$, respectively, which show that the $S^{3}$ is fibered trivially over the $S^{2}$.

Taking the near-horizon limit of $N$ D3-branes at the tip of the cone over $T^{1,1}$, gives the gravity dual, $a d S_{5} \times T^{1,1}$, of the $\mathcal{N}=1$ superconformal gauge field theory described by the superfields $A_{i}, B_{i}$ and the superpotential $W$, as above. The full ten-dimensional warped background metric takes the form [58]:

$$
d s_{10}^{2}=\frac{r^{2}}{L^{2}} d x_{n} d x^{n}+\frac{L^{2}}{r^{2}}\left(d r^{2}+r^{2} d s_{T^{1,1}}^{2}\right) .
$$

Here, the first term in (5) is the usual four-dimensional Minkowski spacetime metric, the second term is the metric on the conifold, given either by (3) or by (4), and $L^{4} \equiv$ $(27 \pi / 4) g_{s} N\left(\alpha^{\prime}\right)^{2}$. In order to have a valid supergravity solution, (5), the number of D3-branes, $N$, has to be large and the string coupling, $g_{s}$, has to be small, so that $g_{s} N \gg 1 ; \alpha^{\prime}=l_{s}^{2}$ denotes the string scale. Here, the dilaton is constant, and the other nontrivial background field is a self-dual $\mathrm{R}-\mathrm{R}$ five-form flux (not written out here).
To add flavored quarks to the pure conformal gauge field theory, one embeds probe flavor $D p$-brane $(s)(p=3,5,7)$ into the gravity dual above. The action of a $D p$-brane is the sum of the DBI and CS actions and takes the general form:

$$
\begin{aligned}
S_{D p}= & -g_{s} T_{p} \int d^{p+1} \xi e^{-\Phi} \sqrt{-\operatorname{det}\left(\gamma_{a b}+\mathscr{F}_{a b}\right)} \\
& +g_{s} T_{p} \int \sum_{p} C_{p+1} \wedge e^{\mathscr{F}_{a b}} .
\end{aligned}
$$

Here, $C_{p+1}$ are the IIB R-R background fields coupling to the $D p$-brane world volume; $\mathscr{F}_{a b}=\mathscr{B}_{a b}+2 \pi \alpha^{\prime} F_{a b}$ is the gauge-invariant field strength with $F_{2}$ the world volume gauge field and $\mathscr{B}_{2}$ the pullback of the NS-NS two-form background field, $B_{2}$, onto the world volume, $\mathscr{B}_{a b}=B_{M N} \partial_{a}$ $X^{M} \partial_{b} X^{N}, \gamma_{a b}=g_{M N} \partial_{a} X^{M} \partial_{b} X^{N}$ the pullback of the tendimensional metric $g_{M N}$ in string frame, and $\Phi=0$. Lastly, $\xi^{a}$ denotes the world volume coordinates, and $T_{p}=$ $\left[(2 \pi)^{p} g_{s}\left(\alpha^{\prime}\right)^{(p+1) / 2}\right]^{-1}$ is the $D p$-brane tension.

2.2. U-like Embedded Probe D7-branes Wrapping ad $S_{5} \times$ $S^{3} \subset a d S_{5} \times T^{1,1}$. As the first example, we consider the Ulike brane embedding configuration of ref. [68], embedding U-like probe D7-branes into the KW gravity dual, corresponding to adding flavored quarks to its dual gauge theory with spontaneous breakdown of the conformal and chiral flavor symmetry. In the KW gravity dual, the probe D7-brane wraps the entire dual spacetime coordinates $\left\{t, x_{i}, r\right\}(i=1,2,3)$ of $a d S_{5}$ in the 01234-directions, and the three-sphere $S^{3} \subset T^{1,1}$ parameterized by the forms $\left\{\Omega_{i}\right\}$ in the 567-directions. Thus, the probe D7-brane wraps the $a d S_{5} \times S^{3} \subset a d S_{5} \times T^{1,1}$ spacetime, and the transverse space is given by the two-sphere $S^{2} \subset T^{1,1}$ parameterized by the coordinates $\theta$ and $\phi$ in the 89 -directions. This all can be summarized and represented by the array:

$$
\begin{array}{lllllllllll} 
& 0 & 1 & 2 & 3 & 4 & 5 & 6 & 7 & 8 & 9 \\
\text { D3 } & \times & \times & \times & \times & & & & & & \\
\text { D7 } & \times & \times & \times & \times & \times & \times & \times & \times & &
\end{array}
$$

Here, we note that, as $w_{i}$ are left-invariant forms, the ansatzes preserves one of the $S U(2)$ factors of the global symmetry group of the conifold, $S U(2) \times S U(2) \times U(1)$. Thus, one may assume that the coordinates $\theta$ and $\phi$ are independent of the $S^{3}$ coordinates. The embedding breaks one of $S U(2)$, but by expanding the action around the solution, it can be shown that contribution from the nontrivial $S^{3}$ shows up only at the second-order fluctuations. Thus, one can assume, in classical sense, that $\theta$ and $\phi$ depend only on the radial coordinate, $r$, of the conifold geometry.

The Kuperstein-Sonnenschein embedding of the D7branes includes two choices. There is one choice where the D7-branes are placed on two separate points of the $S^{2}$, with both of them stretched down to the tip of the conifold at $r=0$ where both the $S^{2}$ and $S^{3}$ shrink to zero size. 
The configuration produced, in this case, is V-like. There is another choice where the D7-branes are placed on the $S^{2}$ with both of them smoothly merged into a single stack somewhere at $r=r_{0}$ above the conifold tip point. The configuration produced, in this case, is $\mathrm{U}$-like. In both of the U-like and V-like configurations, the D7-brane(s) wrap the $a d S_{5} \times S^{3}$ spacetime, as in the array above. However, on the transversal $S^{2}$, here are two different pictures. In the V-like configuration, the D7-branes look like two separate fixed points, while the U-like configuration results an arc along the equator. The position of the two points, setting the position of the D7-branes, depends on $r$ and with them smoothly connected in the midpoint arc at $r=r_{0}$. In the gravity dual, this configuration produces a one-parameter family of D-brane profiles with the parameter $r_{0}$ setting the minimal radial extension of the probe-dual to the IR scale of symmetry breakdown.

By the choice of the world volume fields $\phi=\phi(r)$ and $\theta=\theta(r)$, it is easy to derive the induced world volume metric from (5) in terms of (4) and obtain from (6) the action of the form:

$$
S_{D 7}=-\tilde{T}_{D 7} \int d r d t r^{3} \sqrt{1+\frac{r^{2}}{6}\left(\theta^{\prime 2}+\sin ^{2} \theta \phi^{\prime 2}\right)},
$$

where $\tilde{T}_{D 7}=N_{f} V_{\mathbb{R}^{3}} V_{\mathbb{S}^{3}} T_{D 7}$ with $T_{D 7}=1 /(2 \pi)^{7} g_{s}\left(\alpha^{\prime}\right)^{4}$. The Lagrangian in (8) is $S U(2)$ invariant, and therefore one can restrict motion to the equator of the $S^{2}$ parameterized by $\phi$ and $\theta=\pi / 2$. In writing down the action (8) from (6), one has set $F_{a b}=0$ and noted that since the KW gravity dual contains only $\mathrm{R}-\mathrm{R}$ four-form fluxes, so there is no contribution from the CS part and therefore the D7-brane action is just the DBI action.

The solution of the equation of motion from the action (8) yields a one-parameter family of D7-brane profiles of the form:

$$
\phi(r)=\sqrt{6} r_{0}^{4} \int_{r_{0}}^{r} \frac{d r}{r \sqrt{r^{8}-r_{0}^{8}}}=\frac{\sqrt{6}}{4} \cos ^{-1}\left(\frac{r_{0}}{r}\right)^{4} .
$$

The solution (9) describes two separate branches, a disconnected D7 and an anti-D7-brane pair, including the $\mathrm{V}$-like configuration, when $r_{0}=0$. On contrary, the solution (9) describes a single branch, including the U-like configuration, when the two branches merge at $r=r_{0}>0$. Furthermore, when the configuration is U-like, taking the limit $r \longrightarrow r_{0}$ implies $\phi^{\prime}(r) \longrightarrow \infty$.

The solution (9) contains several important features. First, when the configuration is V-like, it is clear from $d \theta=d \phi=0$ that the induced world volume metric is that of $a d S_{5} \times S^{3}$, and the configuration describes the conformal and chiral symmetric phase. On contrary, when the configuration is U-like, the induced world volume metric has no adS factor and the conformal and chiral flavor symmetry of the dual gauge theory must be broken spontaneously. Second, by taking the asymptotic UV limit, $r \longrightarrow \infty$, (9) describes two constant solutions $\phi_{ \pm}= \pm \sqrt{6} \pi / 8$. This gives an asymptotic UV separation between the branes as $\Delta \phi=\phi_{+}-\phi_{-}=$ $\sqrt{6} \pi / 4$, and an asymptotic expansion as $\phi \simeq \pm \sqrt{6} \pi / 8 \pm \sqrt{6} /$ $4\left(r_{0} / r\right)^{4}+\cdots$. The asymptotic UV separation between the branes is $r_{0}$ independent. The dual gauge field theory is a CFT, in which $r_{0}$ corresponds to a normalizable mode, a vacuum expectation value (VEV). The fact that $r_{0}$ is a modulus, or a flat direction, implies the spontaneous breaking of the conformal symmetry. The expansion reveals that a $\Delta=4$ marginal operator has a VEV fixed by $r_{0}$ as $\left\langle O>\sim r_{0}^{4} /\left(\alpha^{\prime}\right)^{2}\right.$, the fluctuations of which gives the Goldstone boson associated with the conformal symmetry breakdown. Third, the solutions $\phi_{ \pm}$, giving an $r_{0}$-independent UV separation, make the brane antibrane interpretation natural. This is because the brane world volume admits two opposite orientations once the asymptotic points $\phi_{ \pm}$are approached. Fourth, the presence of both the D7 and anti-D7-brane guarantees tadpole cancellation and annihilation of total charge on the transverse $S^{2}$, and it breaks supersymmetry explicitly with the embedding being nonholomorphic.

To this end, one also notes that the above setup cannot be embedded in the $a d S_{5} \times S^{5}$ solution. This is because the $S^{5}$ contains no nontrivial cycle, and therefore the D7-brane will shrink to a point on the $S^{5}$. This problem may be fixed by a specific choice of boundary conditions at infinity, but this turns out to be incompatible with the $U$-shape configuration of interest. In addition, tadpole cancellation by an antiD7-brane is not required, since one has no 2-cycle as in the conifold framework.

2.3. U-like Embedded Probe D5-branes Wrapping ad $S_{3} \times$ $S^{3} \subset a d S_{5} \times T^{1,1}$. As the second example, we consider the Ulike brane embedding configuration of ref. [65], embedding U-like probe D5-branes into the KW gravity dual, corresponding to adding defect flavored quarks to its dual gauge theory with spontaneous breakdown of the conformal and chiral flavor symmetry. In the KW gravity dual, the probe D5-brane wraps some of the dual spacetime coordinates, including $a d S_{3} \subset a d S_{5}$, given by $\left\{t, x_{i}, r\right\}(i=1)$ in the 014directions, and the three-sphere $S^{3} \subset T^{1,1}$ parameterized by the forms $\left\{\Omega_{i}\right\}$ in the 567-directions. Thus, in this example, the probe D5-brane wraps the $a d S_{3} \times S^{3} C$ ad $S_{5} \times T^{1,1}$ spacetime, and the transverse space is given by the two-sphere $S^{2} \subset T^{1,1}$ parameterized by the coordinates $\theta$ and $\phi$ in the 89-directions. This all can be summarized and represented by the array:

$$
\begin{array}{rrrrrrrrrrr} 
& 0 & 1 & 2 & 3 & 4 & 5 & 6 & 7 & 8 & 9 \\
\text { D3 } & \times & \times & \times & \times & & & & & & \\
\text { D5 } & \times & \times & & & \times & \times & \times & \times &
\end{array}
$$

By the choice of the world volume fields similar to the D7-brane above, $\phi=\phi(r)$ and $\theta=$ const., it is easy to 
derive the induced world volume metric from (5) in terms of (4) and obtain from (6) the action of the form:

$$
S_{D 5}=-\tilde{T}_{D 5} \int d r d t r \sqrt{1+\frac{r^{2}}{6} \phi^{\prime 2}}
$$

where $\tilde{T}_{D 5} \equiv N_{f} V_{\mathbb{S}^{3}} T_{D 5}$. Here, we note that the probe D5brane wraps the same $S^{3}$ as the probe D7-brane above, and the Lagrangian density in (11) is the same as the Lagrangian density in (8) modulo $r^{2}$.

The solution of the equation of motion from the action (11) yields a one-parameter family of D5-brane profiles of the form:

$$
\phi(r)=\frac{\sqrt{6}}{2}\left( \pm \frac{\pi}{2} \mp \arctan \left(\frac{r_{0}^{2}}{\sqrt{r^{4}-r_{0}^{4}}}\right)\right) .
$$

The solution (12) has the same general features and satisfies the same boundary conditions, as before. However, in this example, the UV separation is $\Delta \phi=\sqrt{6} \pi / 2$, instead of $\Delta \phi=\sqrt{6} \pi / 4$, in the previous example. Thus, (12) represents another U-shape solution, though this time, dual to dCFT, with the additional quark fields residing in just two out of the four spacetime dimensions, producing a codimension two defects. The expected near boundary behavior of (12) is that of a VEV deformation of a marginal $(\Delta=2)$ operator in one dimension.

2.4. U-like Embedded Probe D5-branes Wrapping adS $S_{4} \times$ $S^{2} \mathrm{CadS}_{5} \times T^{1,1}$. As the third example, we consider the Ulike brane embedding configuration of ref. [65], embedding U-like probe D5-branes alternatively, refs. [66, 67, 85], into the KW gravity dual, corresponding to adding defect flavored quarks to its dual gauge theory with spontaneous breakdown of the conformal and chiral flavor symmetry. In the KW gravity dual, the probe D5-brane wraps some of the dual spacetime coordinates, including $a d S_{4} \subset a d S_{5}$, given by $\left\{t, x_{i}, r\right\} \quad(i=1,2)$ in the 0124-directions, and the two-sphere $S^{2} C T^{1,1}$ parametrized by $\theta_{1}, \phi_{1}$ in the 56directions. Thus, in this example, the probe D5-brane wraps the $a d S_{4} \times S^{2} c a d S_{5} \times T^{1,1}$ spacetime, and the transverse space is given by the three-sphere $S^{3} \subset T^{1,1}$ parametrized by the coordinates $\theta_{2}, \phi_{2}$, and $\psi$ in the 789 -directions. This all can be summarized and represented by the array:

$\begin{array}{lllllllllll} & 0 & 1 & 2 & 3 & 4 & 5 & 6 & 7 & 8 & 9 \\ \text { D3 } & \times & \times & \times & \times & & & & & & \\ \text { D5 } & \times & \times & \times & & \times & \times & \times & & & \end{array}$

By the choice of the world volume fields $\theta_{2}=\pi-\theta_{1}$, $\phi_{1}=\phi_{2}$, and $\psi=\psi(r)$, it is easy to derive the induced world volume metric from the metric (5) in terms of (3) and obtain from (6) the action of the form:

$$
S_{D 5}=-\tilde{T}_{D 5} \int d r d t r^{2} \sqrt{1+\frac{r^{2}}{9} \psi^{\prime 2}}
$$

where $\tilde{T}_{D 5} \equiv N_{f} V_{\mathbb{S}^{2}} T_{D 5}$. Here, we note that the probe D5brane wraps this time the $S^{2}$, unlike in the previous example, and the Lagrangian density in (14) is not the same as the Lagrangian density in (11).

The solution of the equation of motion from the action (14) yields a one-parameter family of D5-brane profiles of the form:

$$
\psi(r)= \pm \frac{\pi}{2} \mp \arcsin \left(\frac{r_{0}^{3}}{r^{3}}\right) .
$$

The solution (15) has the same of the general features and meets the same boundary conditions, as before. However, in this example, the UV separation is given by $\Delta \psi=$ $\psi_{+}-\psi_{-}=\pi / 2-(-\pi / 2)=\pi$, with both $\psi_{ \pm}$corresponding to supersymmetric D5-brane embedding and preserving different supersymmetries. Thus, (15) represents another Ushape solution, though this time, supersymmetric and dual to dCFT, with the additional quark fields residing in three out of the four spacetime dimensions, producing a codimension one defect. The expected near boundary behavior of (15) is that of a VEV deformation of a marginal $\Delta=2$ operator.

2.5. U-like Embedded Probe D3-branes Wrapping ad $S_{2} \times$ $S^{2} \mathrm{CadS}_{5} \times T^{1,1}$. As the final example, we consider the Ulike brane embedding configuration of ref. [65], embedding U-like probe D3-branes into the KW gravity dual, corresponding to adding defect flavored quarks to its dual gauge theory with spontaneous breakdown of the conformal and chiral flavor symmetry. In the KW gravity dual, the probe D3-brane wraps some of the dual spacetime coordinates, including $a d S_{2} \mathrm{CadS}_{5}$, given by $\{t, r\}$ in the 04-directions, and the two-sphere $S^{2} \subset T^{1,1}$ parameterized by $\theta_{1}, \phi_{1}$ in the 56-directions. Thus, the probe D3-brane wraps the $a d S_{2} \times$ $S^{2}$ spacetime, and the transverse space is given by the threesphere $S^{3} \subset T^{1,1}$ parameterized by the coordinates $\theta_{2}, \phi_{2}$, and $\psi$ in the 789-directions. This all can be summarized and represented by the array:

$\begin{array}{lllllllllll} & 0 & 1 & 2 & 3 & 4 & 5 & 6 & 7 & 8 & 9 \\ \text { D3 } & \times & \times & \times & \times & & & & & & \\ \text { D3 } & \times & & & & \times & \times & \times & & \end{array}$

By the choice of the world volume fields similar to the probe D5-brane above, $\phi=\theta=$ const. and $\psi=\psi(r)$, it is easy 
to derive the induced world volume metric from (5) in terms of (3) and obtain from (6) the action of the form:

$$
S_{D 3}=-\tilde{T}_{D 3} \int d r d t \sqrt{1+\frac{r^{2}\left(\psi^{\prime}\right)^{2}}{9}},
$$

where $\tilde{T}_{D 3}=N_{f} V_{\mathbb{S}^{2}} T_{D 3}$. We also note that the Lagrangian density in (17) is, up to the radial factor, $r^{2}$, the same as the density of the probe D5-brane in (14).

The solution of the equation of motion from the action (17) yields a one-parameter family of D3-brane profiles of the form:

$$
\psi(r)=\mp \frac{3 \pi}{2} \pm 3 \arctan \left(\frac{r_{0}}{\sqrt{r^{2}-r_{0}^{2}}}\right)
$$

The solution (18) has the same general features and satisfies the same boundary conditions, as before. However, in this example, the UV separation is $\Delta \psi=3 \pi$, whereby the brane never wraps the internal cycle more than once, as $\psi \in[0,4 \pi]$. Thus, (18) represents another U-shape solution dual to dCFT, with the additional quark fields being "quantum mechanical," depending only on time and not on space. The expected near boundary behavior of (18) is that of a VEV deformation of a marginal $(\Delta=1)$ operator in one dimension.

\section{Induced Metric and Temperature on U-like Embedded Probes}

In this section, we first modify the Kuperstein-Sonnenschein and BKS models reviewed above, by turning on additional spin on the U-like embedded probes dual to exciting Rcharge in the flavor sector of the CFT and dCFTs with spontaneous conformal and chiral flavor symmetry breakdown. We do this by noting, in general, that global rotational symmetry implies conserved angular momentum. This means, in all our examples below, that the RHS of our field equations vanishes because the time derivative of our ansatzes solutions is constant, making our solutions stationary. We also note that on the conifold background (see Sec. 2) with global symmetry $S U(2) \times S U(2) / U(1)$ parametrized by $(\psi, \phi, \theta)$, such ansäze for the transverse world-volume scalars constitute exclusively the directions $\psi \in[0,4 \pi]$ and $\phi \in[0,2 \pi]$ consistent with rotations; with the other "oscillatory" directions $\theta \in[0, \pi]$ for the transverse scalars fixed. We then consider the simplest case of single field ansäze for the world-volume scalars, with world-volume gauge fields turned off, $F_{a b}=0$, and compute the related induced world volume metrics and temperatures on the U-like embedded rotating probes.

3.1. Induced Metric and Temperature on U-like Embedded Probe D7-brane Wrapping ad $S_{5} \times S^{3} \subset a d S_{5} \times T^{1,1}$ and Spinning about $S^{2} \subset T^{1,1}$. As the first and warm-up example in our study, we consider the U-like probe D7-brane model wrapping $a d S_{5} \times S^{3}$ in $a d S_{5} \times T^{1,1}$ reviewed in Sec. 2, and turn on additional spin for the probe, following closely [55]. We include additional spin degrees of freedom, dual to finite R-charge chemical potential, by allowing in our system the D7-brane rotate in the $\phi$ direction of the transverse $S^{2} \subset T^{1,1}$ with conserved angular momentum. Thus, in our setup, we allow $\phi$ to have time-dependence, such that $\dot{\phi}(r, t)=\omega=$ const., with $\omega$ denoting the angular velocity of the probe. This way, we construct rotating solutions. Hence, the world-volume field is given by $\phi(r, t)$, with other directions fixed.

In this section, therefore, we will study U-like embedded probe D7-branes spinning with an angular frequency $\omega$. Thus, we will consider the ansatzes for the D7-brane worldvolume field of the form $\phi(r, t)=\omega t+f(r)$, and $F_{a b}=0$. Using this ansatzes and the metric (5) in terms of (4), it is easy to derive the components of the induced worldvolume metric on the D7-brane, $g_{a b}^{D 7}$, and compute the determinant, det $g_{a b}^{D 7}$, resulting the DBI action (6), as:

$$
S_{D 7}=-\tilde{T}_{D 7} \int d r d t r^{3} \sqrt{1-\frac{L^{4} \dot{\phi}^{2}}{6 r^{2}}+\frac{r^{2}\left(\phi^{\prime}\right)^{2}}{6}} .
$$

Here, we note that by setting $\dot{\phi}=\omega=0$, our action (19) reduces to that of the probe D7-brane action in the Kuperstein-Sonnenschein model, (8). As in the Kuperstein-Sonnenschein model reviewed in Sec. 2, we restrict brane motion to the $\phi$-direction of the transverse $S^{2}$ sphere and fix other directions constant. Thus, in our setup we let, in addition, the probe rotate about the $S^{2}$.

From (19), the D7-brane equation of motion and solution read:

$$
\begin{gathered}
\frac{\partial}{\partial r}\left[\frac{r^{5} \phi^{\prime}}{\sqrt{1+\left(r^{2}\left(\phi^{\prime}\right)^{2} / 6\right)-L^{4} \dot{\phi}^{2} / 6 r^{2}}}\right] \\
=\frac{\partial}{\partial t}\left[\frac{L^{4} r \dot{\phi}}{\sqrt{1+\left(r^{2}\left(\phi^{\prime}\right)^{2} / 6\right)-L^{4} \dot{\phi}^{2} / 6 r^{2}}}\right] . \\
\phi(r, t)=\omega t+f(r), \quad f(r)=\sqrt{6} r_{0}^{4} \int_{r 0}^{r} \frac{d r}{r} \sqrt{\frac{1-L^{4} \bar{\omega}^{2} / r^{2}}{r^{8}-r_{0}^{8}}} .
\end{gathered}
$$

Here, we set $\bar{\omega}=\omega / \sqrt{6}$ and note that when $\omega=0$, our solution (21) integrates to that of probe D7-brane in the Kuperstein-Sonnenschein model, [68], reviewed in Sec. 2, with the probe wrapping $a d S_{5} \times S^{3}$ in $a d S_{5} \times T^{1,1}$. The solution $(21)$ is parameterized by $\left(r_{0}, \omega\right)$ and describes probe D7-brane motion with angular velocity $\omega$ about the 
transverse $S^{2} \subset T^{1,1}$, starting and ending up at the boundary. The probe descends from the UV boundary at infinity to the minimal extension $r_{0}$ dual to the IR scale where it bends back up the boundary. We also note that inspection of (21) shows that in the limit $r \longrightarrow$ large, the $\omega$-dependent term is subleading and therefore the behavior of $d f(r) / d r=f_{r}(r)$ does not depend on $\omega$. This means that in the large radii limit the solution $f(r)$ in (21) gives the world-volume field $\phi(r)$ of the Kuperstein-Sonnenschein model (see Sec. 2) with the boundary values $\phi_{ \pm}$in the asymptotic UV limit, $r \longrightarrow \infty$, independent from $\omega$. Nonetheless, we note that in the IR limit, $r \longrightarrow$ small, the $\omega$-dependent term becomes large, and thus the behavior of $f_{r}(r)$ may depend on $\omega$. However, inspection of (21) shows that one can always choose $\omega$ so that in the IR the behavior of $f_{r}(r)$ with $\omega>0$ is comparable to that of with $\omega=0$. This means that for certain $\omega>0$ in the small radii limit in the IR the behavior of $f_{r}(r)$ does compare to that of $\phi^{\prime}(r)=d \phi / d r$ in the Kuperstein-Sonnenschein model (see Sec. 2), with $\phi^{\prime}(r) \longrightarrow \infty$ in the IR limit $r \longrightarrow$ $r_{0}$, consistent with U-like embedding (see Figure 1).

To derive the induced metric on the D7-brane, we put the rotating solution (21) into the background metric (5) in terms of (4) and obtain:

$$
\begin{aligned}
d s_{D 7}^{2}= & -\frac{1}{3 L^{2}}\left(3 r^{2}-L^{4} \omega^{2}\right) d t^{2} \\
& +\frac{L^{2}}{r^{2}}\left[\frac{3 r^{2}\left(r^{8}-r_{0}^{8}\right)+r_{0}^{8}\left(6 r^{2}-L^{4} \omega^{2}\right)}{3 r^{2}\left(r^{8}-r_{0}^{8}\right)}\right] d r^{2} \\
& +\frac{2 L^{2} \omega r_{0}^{4}}{3 r^{2}} \sqrt{\frac{6 r^{2}-L^{4} \omega^{2}}{r^{8}-r_{0}^{8}}} d r d t \\
& +\frac{r^{2}}{L^{2}}\left(d x^{2}+d y^{2}+d z^{2}\right)+\frac{L^{2}}{3}\left[\frac{1}{2}\left(\Omega_{1}^{2}+\Omega_{2}^{2}\right)+\frac{1}{3} \Omega_{3}^{2}\right] \\
& -\frac{L^{2}}{3}\left[\omega \Omega_{1} d t+\frac{r_{0}^{4}}{r^{2}} \sqrt{\frac{6 r^{2}-L^{4} \omega^{2}}{r^{8}-r_{0}^{8}}} \Omega_{1} d r\right] .
\end{aligned}
$$

Here, we note that by setting $\omega=0$, our induced worldvolume metric (22) reduces to that of the Kuperstein-Sonnenschein model, [68], reviewed in Sec. 2. In this case, for $r_{0}=0$, the induced world-volume metric is that of $a d S_{5} \times S^{3}$ , and the dual gauge theory describes the conformal and chiral symmetric phase. On the contrary, for $r_{0}>0$, the induced world volume metric has no adS factor, and the conformal invariance of the dual gauge theory must be broken in such case. In order to find the world-volume horizon and Hawking temperature, we first eliminate the relevant cross term. To eliminate the relevant cross-term in (22), we consider a coordinate transformation:

$$
\tau=t-\omega L^{4} r_{0}^{4} \int \frac{d r\left(6 r^{2}-L^{4} \omega^{2}\right)^{1 / 2}}{r^{2}\left(3 r^{2}-L^{4} \omega^{2}\right)\left(r^{8}-r_{0}^{8}\right)^{1 / 2}} .
$$

The induced metric on the rotating D7-brane(s) then takes the form:

$$
\begin{aligned}
d s_{D 7}^{2}= & -\frac{\left(3 r^{2}-L^{4} \omega^{2}\right)}{3 L^{2}} d \tau^{2} \\
& +\frac{L^{2}}{r^{2}}\left[\frac{\left(3 r^{2}-L^{4} \omega^{2}\right)\left(r^{8}-r_{0}^{8}\right)+r_{0}^{8}\left(6 r^{2}-L^{4} \omega^{2}\right)}{\left(3 r^{2}-L^{4} \omega^{2}\right)\left(r^{8}-r_{0}^{8}\right)}\right] d r^{2} \\
& +\frac{r^{2}}{L^{2}}\left(d x^{2}+d y^{2}+d z^{2}\right)+\frac{L^{2}}{3}\left[\frac{1}{2}\left(\Omega_{1}^{2}+\Omega_{2}^{2}\right)+\frac{1}{3} \Omega_{3}^{2}\right] \\
& -\frac{L^{2}}{3}\left[\omega \Omega_{1} d \tau+\frac{3 r_{0}^{4}}{3 r^{2}-L^{4} \omega^{2}} \sqrt{\frac{6 r^{2}-L^{4} \omega^{2}}{r^{8}-r_{0}^{8}}} \Omega_{1} d r\right] .
\end{aligned}
$$

Here, we note that for $r_{0}=0$ the induced world-volume metric (24) has no horizon solving $-g_{\tau \tau}=g^{r r}=0$, and thus not given by the black hole geometry. On the contrary, for $r_{0}>0$, the induced world-volume horizon solves the equation:

$$
H(r)=r^{2}\left(r^{8}-r_{0}^{8}\right)\left(3 r^{2}-L^{4} \omega^{2}\right)=0 .
$$

Eq. (25) contains two real positive definite zeros. The thermal horizon of the induced world-volume black hole geometry is given by the zero $3 r_{H}^{2}-L^{4} \omega^{2}=0$, with the horizon varying continuously with varying the angular velocity $\omega$, as expected. Eq. (25) also shows that the horizon must grow from the minimal extension $r_{0} \neq 0$-dual to the IR scale of symmetry breakdown with increasing the angular velocity $\omega$. We thus conclude at this point that when $r_{0}$ is positive definite $\left(r_{0}>0\right)$ and spin is turned on $(\omega>0)$, the induced world-volume metric on the rotating probe D7-brane wrapping $a d S_{5} \times S^{3}$ in $a d S_{5} \times T^{1,1}$ admits a thermal horizon growing continuously with increasing the angular velocity.

The Hawking temperature on the probe D7-brane can be found from the induced world-volume metric (24) in the form:

$$
T_{H ; D 7}=\left.\frac{\left(g^{r r}\right)^{\prime}}{4 \pi}\right|_{r=r_{H}}=\frac{3 r_{H}^{3}\left(r_{H}^{8}-r_{0}^{8}\right)}{2 \pi L^{2} r_{0}^{8}\left[6 r_{H}^{2}-L^{4} \omega^{2}\right]}=\frac{r_{H}\left(r_{H}^{8}-r_{0}^{8}\right)}{2 \pi L^{2} r_{0}^{8}} .
$$

Here, we note from (26) that when the world volume horizon is at the minimal radial extension-dual to the IR scale, $r_{H}=r_{0}$, the Hawking temperature of the world volume black hole geometry is identically zero, $T_{H ; D 7}=0$. We note, however, that the world-volume Hawking temperature $T_{H ; D 7}$ (26) increases monotonically with increasing the angular velocity $\omega$, by which the world volume horizon size $r_{H}$ grows. In this case, changing $r_{0}$ in (26) changes the scale, but not the functional form and behavior of the temperature (see Figure 1). 

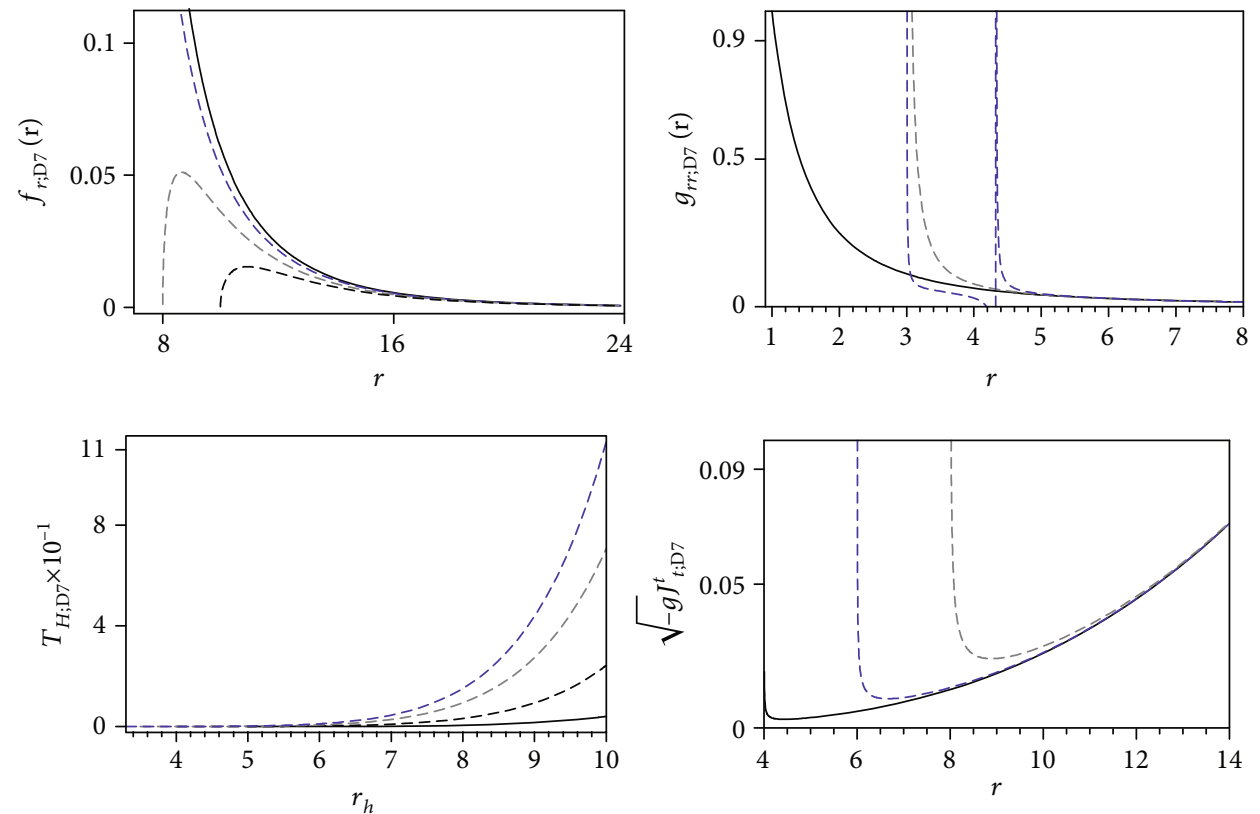

FIGURE 1: The behavior of the derivative of the D7-brane world volume field with respect to $r$ with $L=1, r_{0}=7, \omega=0$ (black-solid), $\omega=5$ (blue-dashed), $\omega=8$ (gray-dashed), and $\omega=10$ (black-dashed) (up-left). The behavior of the $g_{r r}$ component of the D7-brane induced world volume metric with $L=1, r_{0}=0, \omega=0$ (black-solid), $r_{0}=3, \omega=0$ (gray-dashed), and $r_{0}=3, \omega=7.5$ (blue-dashed) (up-right). The behavior of the world volume Hawking temperature of the probe D7-brane with $L=10$ and $r_{0}=5$ (black-solid), $r_{0}=4$ (black-dashed), $r_{0}=3.5$ (gray-dashed), $r_{0}=3$ (blue-dashed) (down-left). The behavior of the energy density of the probe D7-brane with $L=\omega=1$ and $r_{0}=4$ (black-solid), $r_{0}=6$ (blue-dashed), and $r_{0}=8$ (gray-dashed) (down-right).

We further notice that by taking into account the backreaction of the above solution to the KW gravity dual, $a d S_{5} \times T^{1,1}$, one accordingly awaits the D7-brane to form a mini black hole in KW, in the probe limit. Thus, the dual gauge theory couples to a thermal object and describes a locally thermal vacuum. In the KW example here, the configuration is dual to $\mathcal{N}=1$ gauge theory coupled to a flavor with spontaneous conformal and chiral flavor symmetry breakdown. As the gauge theory itself is at zero temperature, while its flavor sector is at finite temperature (26), we conclude that the configuration describes nonequilibrium steady state in the gauge theory conformal and chiral symmetry breakdown. However, as we shall show below, the energy from the flavor sector will in the end dissipate into the gauge theory conformal and chiral flavor symmetry breakdown.

So far, in this example, we neglected the backreaction of the D7-brane to the KW gravity dual, as we worked in the probe limit. It is useful to observe the extend this is justifiable. To this end, we derive the components of the energy-stress tensor of our D-brane system. We first note that the energy-stress tensor and its equation of motion take the form:

$$
J_{b}^{a}=\frac{2}{\sqrt{-g}} \frac{\delta S}{\delta g_{a c}} g_{a c}, \quad \nabla_{a} J_{b}^{a}=\partial_{a}\left(J_{t}^{a} \sqrt{-g}\right)=0 .
$$

Here, we note that the LHS of the equation of motion in (27) has been reduced to $\partial_{a}\left(T_{t}^{a} \sqrt{-g}\right)$ for stationary solutions constructed above. This implies the conserved energy of the form:

$$
E=\int d r \sqrt{-g} J_{t}^{t}
$$

We then note that for our rotating D7-brane, the components of the energy-stress tensor in (27) take the form:

$$
\begin{aligned}
\sqrt{-g} J_{t ; D 7}^{t} \equiv & \frac{\tilde{T}_{D 7} r^{3}\left(1+r^{2}\left(\phi^{\prime}\right)^{2} / 6\right)}{\sqrt{1+r^{2}\left(\phi^{\prime}\right)^{2} / 6-L^{4} \dot{\phi}^{2} / 6 r^{2}}} \\
& =\frac{\tilde{T}_{D 7}\left(r^{10}-L^{4} \bar{\omega}^{2} r_{0}^{8}\right)}{\sqrt{\left(r^{8}-r_{0}^{8}\right)\left(r^{6}-L^{4} \bar{\omega}^{2} r^{4}\right)}},
\end{aligned}
$$

$$
\begin{aligned}
\sqrt{-g} r_{r ; D 7}^{r} & \equiv-\frac{\tilde{T}_{D 7} r^{3}\left(1-L^{4} \dot{\phi}^{2} / 6 r^{2}\right)}{\sqrt{1+r^{2}\left(\phi^{\prime}\right)^{2} / 6-L^{4} \dot{\phi}^{2} / 6 r^{2}}} \\
& =-\tilde{T}_{D 7}\left(r^{2}-L^{4} \bar{\omega}^{2}\right) \sqrt{\frac{r^{8}-r_{0}^{8}}{r^{6}-L^{4} \bar{\omega}^{2} r^{4}}}
\end{aligned}
$$




$$
\sqrt{-g} J_{t ; D 7}^{r} \equiv \frac{\tilde{T}_{D 7} r^{5} \dot{\phi} \phi^{\prime}}{\sqrt{1+r^{2}\left(\phi^{\prime}\right)^{2} / 6-L^{4} \dot{\phi}^{2} / 6 r^{2}}}=\tilde{T}_{D 7} r_{0}^{4} \omega
$$

Using (29)-(31), we can derive the total energy and energy flux of the D-brane system. The total energy of the D7-brane in the above configuration is given by:

$$
E=\tilde{T}_{D 7} \int_{r_{0}}^{\infty} \frac{d r\left(r^{10}-L^{4} \bar{\omega}^{2} r_{0}^{8}\right)}{r^{4} \sqrt{\left(r^{8}-r_{0}^{8}\right)\left(r^{6}-L^{4} \bar{\omega}^{2} r^{4}\right)}} .
$$

It is straightforward to see from (32) that when $r \longrightarrow r_{0}$, the energy density of the flavor brane, given by (29), becomes very large and blows up precisely at the minimal extension $r=r_{0}$-dual the IR scale of conformal and chiral flavor symmetry breakdown-independent from the presence of the world volume angular velocity. We also note that changing $r_{0}$ merely shifts the point where the energy density blows up but leaves the behavior of the density unchanged (see Figure 1). Thus, we conclude that at the IR scale, $r=r_{0}$, the backreaction of the D7-brane to the gravity dual metric is nonnegligibly large and forms a black hole centered at the IR scale $r=r_{0}$ in the bulk. The black hole size should grow as the energy gets pumped into it from the D7-brane steadily. We note that the energy density does not transform as a scalar under coordinate transformations. In order to obtain the energy flux, we compute the time evolution of the total energy by using the components of the energy-stress tensor (29)-(31) and the conserved energy (28), corresponding to our stationary solutions-consistent with global rotational symmetry. We then note that when the minimal radial extension-dual to the IR scale of symmetry breakdown is positive definite $\left(r_{0}>0\right)$ and spin is turned on $(\omega>0)$; the component (31) is nonvanishing, and hence, we compute from (28) the time evolution of the total energy as:

$$
\begin{aligned}
\dot{E}_{D 7} & =\frac{d}{d t} \int d r \sqrt{-g} J_{t ; D 7}^{t}=\int d r \partial_{r}\left(\sqrt{-g} J_{t ; D 7}^{r}\right) \\
& =\left.\sqrt{-g} J_{t ; D 7}^{r}\right|_{r=r_{0}} ^{\infty}=\tilde{T}_{D 7} r_{0}^{4} \omega^{2}-\tilde{T}_{D 7} r_{0}^{4} \omega^{2}=0 .
\end{aligned}
$$

Relation (33) shows that the energy per unit time injected at the UV boundary $r=\infty$ by some external system equals the energy dissipated from the IR into the bulk, despite the total energy is time-independent. Thus, the energy will dissipate from the brane into the bulk and will form a black hole, centered at the IR scale $r=$ $r_{0}$. We note that when $\omega=0$, relation (33) is trivially satisfied, on contrary with the case $\omega \neq 0$, corresponding to our stationary solutions-consistent with global rotational symmetry. Thus, by this flow of energy from the probe to the bulk, we conclude by duality that the energy from the flavor sector will eventually dissipate into the gauge theory conformal and chiral flavor symmetry breakdown. To see this external injection of energy in our stationary rotating solution, we may introduce UV and IR cutoffs to the D7-brane solution, such that it extends from $r=$ $r_{\mathrm{IR}}=r_{0}$ to $r=r_{\mathrm{UV}} \gg r_{\mathrm{IR}}$. It is clear from (31) that at both $r_{\mathrm{IR}}$ and $r=r_{\mathrm{UV}}$, we have $J_{t}^{r}>0$. This shows the presence of equal energy flux incoming from $r=r_{\mathrm{UV}}$ and outgoing at $r=r_{\mathrm{IR}}$ at which the energy gets not reflected back, but its backreaction will nucleate a black hole intaking the injected energy.

\subsection{Induced Metric and Temperature on U-like Embedded} Probe D5-brane Wrapping ad $S_{3} \times S^{3} \subset a d S_{5} \times T^{1,1}$ and Spinning about $S^{2} \subset T^{1,1}$. As the second example in our study, we consider the U-like probe D5-brane model wrapping ad $S_{3} \times S^{3}$ in $a d S_{5} \times T^{1,1}$ reviewed in Sec. 2, and turn on additional spin for the probe. We include additional spin degrees of freedom, dual to finite $\mathrm{R}$-charge chemical potential, by allowing in our system the D5-brane rotate in the $\phi$ direction of the transverse $S^{2} \subset T^{1,1}$ with conserved angular momentum. Thus, in our setup, we allow $\phi$ to have time-dependence, such that $\dot{\phi}(r, t)=\omega=$ const., with $\omega$ denoting the angular velocity of the probe. This way, we construct rotating solutions. Hence, the world-volume field is given by $\phi(r, t)$, with other directions fixed.

In this section, therefore, we will study U-like embedded probe D5-branes spinning with an angular frequency $\omega$. Thus, we will consider the ansatzes for the D5-brane worldvolume field of the form $\phi(r, t)=\omega t+f(r)$ and $F_{a b}=0$. Using this ansatzes and the metric (5) in terms of (4), it is easy to derive the components of the induced worldvolume metric on the D5-brane, $g_{a b}^{D 5}$, and compute the determinant, det $g_{a b}^{D 5}$, resulting the DBI action (6), as:

$$
S_{D 5} \simeq-T_{D 5} \int d r d t r \sqrt{1+\frac{r^{2}\left(\phi^{\prime}\right)^{2}}{6}-\frac{L^{4} \dot{\phi}^{2}}{6 r^{2}}} \text {. }
$$

Here, we note that by setting $\dot{\phi}=\omega=0$, our action (34) reduces to that of the probe D5-brane action in the BKS model, (8). As in the BKS model reviewed in Sec. 2, we restrict brane motion to the $\phi$-direction of the transverse $S^{2}$ sphere and fix other directions constant. Thus, in our setup we let, in addition, the probe rotate about the $S^{2}$, as before.

From (34), the D5-brane equation of motion and solution read:

$$
\begin{gathered}
\frac{\partial}{\partial r}\left[\frac{r^{3} \phi^{\prime}}{\sqrt{1+\left(r^{2}\left(\phi^{\prime}\right)^{2} / 6\right)-L^{4} \dot{\phi}^{2} / 6 r^{2}}}\right] \\
=\frac{\partial}{\partial t}\left[\frac{L^{4} \dot{\phi}}{r \sqrt{1+\left(r^{2}\left(\phi^{\prime}\right)^{2} / 6\right)-L^{4} \dot{\phi}^{2} / 6 r^{2}}}\right],
\end{gathered}
$$



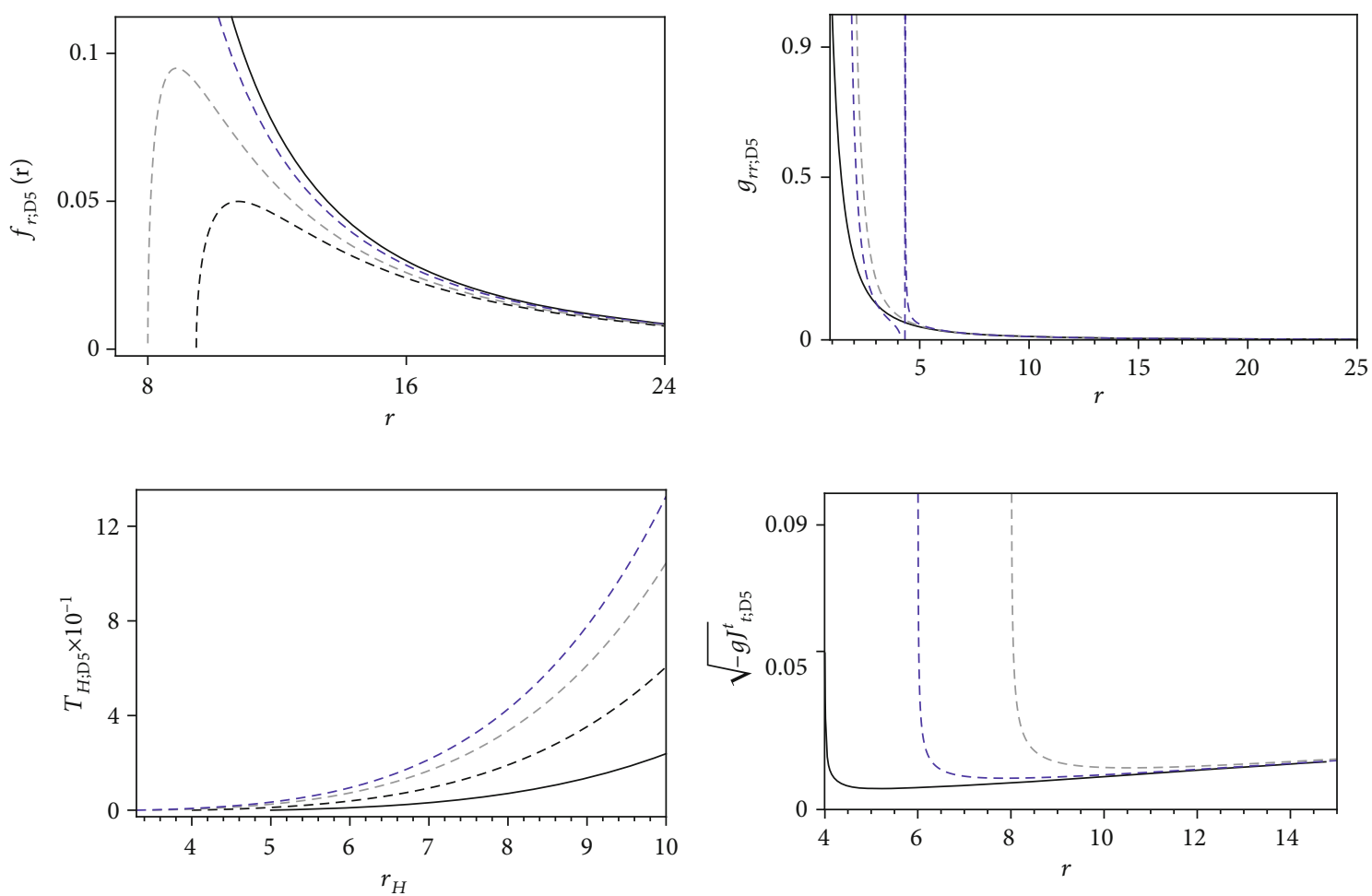

Figure 2: The behavior of the derivative of the D5-brane world volume field with respect to $r$ with $L=1, r_{0}=7, \omega=0$ (black-solid), $\omega=5$ (blue-dashed), $\omega=8$ (gray-dashed), and $\omega=10$ (black-dashed) (up-left). [Up-Right] The behavior of the $g_{r r}$ component of the D5-brane induced world volume metric with $L=1, r_{0}=0, \omega=0$ (black-solid), $r_{0}=1.5, \omega=0$ (gray-dashed), and $r_{0}=1.5, \omega=7.5$ (blue-dashed). [Down-Left] The behavior of the world volume Hawking temperature of the probe D5-brane with $r_{0}=5$ (black-solid), $r_{0}=4$ (blackdashed), $r_{0}=3.5$ (gray-dashed), $r_{0}=3$ (blue-dashed), and $L=10$. [Down-Right] The behavior of the energy density of the probe D5-brane with $L=\omega=1$ and $r_{0}=4$ (black-solid), $r_{0}=6$ (blue-dashed), and $r_{0}=8$ (gray-dashed).

$$
\phi(r, t)=\omega t+f(r), f(r)=\sqrt{6} r_{0}^{2} \int_{r_{0}}^{r} \frac{d r}{r} \sqrt{\frac{1-L^{4} \bar{\omega}^{2} / r^{2}}{r^{4}-r_{0}^{4}}} .
$$

Here, we set $\bar{\omega}=\omega / \sqrt{6}$ and note that when $\omega=0$, our solution (36) integrates to that of probe D5-brane in the BKS model, [65], reviewed in Sec. 2, with the probe wrapping $a d S_{3} \times S^{3}$ in $a d S_{5} \times T^{1,1}$ (see Eq. (12)). The solution (36) is parametrized by $\left(r_{0}, \omega\right)$ and describes probe D5-brane motion with angular velocity $\omega$ about the transverse $S^{2} C$ $T^{1,1}$, starting and ending up at the boundary. The probe descends from the UV boundary at infinity to the minimal extension $r_{0}$-dual to the IR scale of symmetry breakdown where it bends back up the boundary. We also note that inspection of (36) shows that in the limit $r \longrightarrow$ large the $\omega$-dependent term is subleading and therefore the behavior of $d f(r) / d r=f_{r}(r)$ does not depend on $\omega$. This shows that in the large radii limit, the solution $f(r)$ in (36) gives the world volume field $\phi(r)$ of the BKS model (see Sec. 2) with the boundary values $\phi_{ \pm}$in the asymptotic UV limit, $r \longrightarrow \infty$. Nonetheless, we note that in the IR limit, i.e., when $r \longrightarrow$ small, the $\omega$-dependent term becomes large, and therefore the behavior of $f_{r}(r)$ may depend on $\omega$. However, inspection of (36) show that one can always fix $L$ and regulate $\omega$ so that in the IR, the behavior of $f_{r}(r)$ with $\omega>0$ is comparable to that of with $\omega=0$. This means that for certain $\omega>0$ in the small radii limit in the IR the behavior of $f_{r}(r)$ does compare to that of $\phi^{\prime}(r)=d \phi / d r$ in the BKS (see Sec. 2), with $\phi^{\prime}(r) \longrightarrow \infty$ in the IR limit $r \longrightarrow r_{0}$, consistent with U-like embedding (see Figure 2).

To derive the induced metric on the D5-brane, we put the rotating solution (36) into the background metric (5) in terms of (4) and obtain:

$$
\begin{aligned}
d s_{D 5}^{2}= & -\frac{\left(3 r^{2}-L^{4} \omega^{2}\right)}{3 L^{2}} d t^{2} \\
& +\frac{L^{2}}{r^{2}}\left[\frac{3 r^{2}\left(r^{4}-r_{0}^{4}\right)+r_{0}^{4}\left(6 r^{2}-L^{4} \omega^{2}\right)}{3 r^{2}\left(r^{4}-r_{0}^{4}\right)}\right] d r^{2} \\
& +\frac{2 L^{2} \omega r_{0}^{2}}{3 r^{2}} \sqrt{\frac{6 r^{2}-L^{4} \omega^{2}}{r^{4}-r_{0}^{4}}} d r d t+\frac{r^{2}}{L^{2}} d x^{2} \\
& +\frac{L^{2}}{3}\left[\frac{1}{2}\left(\Omega_{1}^{2}+\Omega_{2}^{2}\right)+\frac{1}{3} \Omega_{3}^{2}\right. \\
& \left.+\omega \Omega_{1} d t-\frac{r_{0}^{2}}{r^{2}} \sqrt{\frac{6 r^{2}-L^{4} \omega^{2}}{r^{4}-r_{0}^{4}}} \Omega_{1} d r\right]
\end{aligned}
$$

Here, we note that by setting $\omega=0$, our induced world volume metric (37) reduces to that of the BKS model, [65], reviewed in Sec. 2. In this case, for $r_{0}=0$, the induced world 
volume metric is that of $a d S_{3} \times S^{3}$, and the dual gauge theory describes the conformal and chiral symmetric phase. On the contrary, for $r_{0}>0$, the induced world volume metric has no adS factor and the conformal invariance of the dual gauge theory must be broken in such case. In order to find the world volume horizon and Hawking temperature, we first eliminate the relevant cross term. To eliminate the relevant cross-term in (37), we consider a coordinate transformation:

$$
\tau=t-\omega L^{4} r_{0}^{2} \int \frac{d r\left(6 r^{2}-L^{4} \omega^{2}\right)^{1 / 2}}{r^{2}\left(3 r^{2}-L^{4} \omega^{2}\right)\left(r^{4}-r_{0}^{4}\right)^{1 / 2}} .
$$

The induced metric on the rotating D5-brane, (37), then takes the form:

$$
\begin{aligned}
d s_{D 5}^{2}= & -\frac{\left(3 r^{2}-L^{4} \omega^{2}\right)}{3 L^{2}} d \tau^{2} \\
& +\frac{L^{2}}{r^{2}}\left[\frac{\left(3 r^{2}-L^{4} \omega^{2}\right)\left(r^{4}-r_{0}^{4}\right)+r_{0}^{8}\left(6 r^{2}-L^{4} \omega^{2}\right)}{\left(3 r^{2}-L^{4} \omega^{2}\right)\left(r^{4}-r_{0}^{4}\right)}\right] d r^{2} \\
& +\frac{r^{2}}{L^{2}} d x^{2}+-\frac{L^{2}}{3}\left[\frac{1}{2}\left(\Omega_{1}^{2}+\Omega_{2}^{2}\right)+\frac{1}{3} \Omega_{3}^{2}\right] \\
& -\frac{L^{2}}{3}\left[\omega \Omega_{1} d \tau+\frac{3 r_{0}^{2}}{3 r^{2}-L^{4} \omega^{2}} \sqrt{\frac{6 r^{2}-L^{4} \omega^{2}}{r^{4}-r_{0}^{4}}} \Omega_{1} d r\right]
\end{aligned}
$$

Here, we note that for $r_{0}=0$ the induced world volume metric (39) has no horizon solving $-g_{\tau \tau}=g^{r r}=0$, and thus not given by the black hole geometry. On the contrary, for $r_{0}>0$, the induced world volume horizon solves the equation:

$$
H(r)=r^{2}\left(r^{4}-r_{0}^{4}\right)\left(3 r^{2}-L^{4} \omega^{2}\right)=0
$$

Eq. (40) contains two real positive definite zeros. The thermal horizon of the induced world volume black hole geometry is given by the zero $3 r_{H}^{2}-L^{4} \omega^{2}=0$, with the horizon varying continuously with varying the angular velocity $\omega$, as expected. Eq. (40) also shows that the horizon must grow from the minimal extension $r_{0} \neq 0-$ dual to the IR scale of symmetry breakdown with increasing the angular velocity $\omega$, as before. We thus conclude at this point that when $r_{0}$ is positive definite $\left(r_{0}>0\right)$ and spin is turned on $(\omega>0)$, the induced world volume metric on the rotating probe D5-brane wrapping $a d S_{3} \times S^{3}$ in $a d S_{5} \times T^{1,1}$ admits a thermal horizon growing continuously with increasing the angular velocity.

The Hawking temperature on the probe D5-brane can be found from the induced world-volume metric (39) in the form:

$$
T_{H ; D 5}=\left.\frac{\left(g^{r r}\right)^{\prime}}{4 \pi}\right|_{r=r_{H}}=\frac{3 r_{H}^{3}\left(r_{H}^{4}-r_{0}^{4}\right)}{2 \pi L^{2} r_{0}^{4}\left[6 r_{H}^{2}-L^{4} \omega^{2}\right]}=\frac{r_{H}\left(r_{H}^{4}-r_{0}^{4}\right)}{2 \pi L^{2} r_{0}^{4}}
$$

Here, we note that from (41) that when the world volume horizon is at the minimal radial extension $r_{H}=$ $r_{0}$-dual to the IR scale, the Hawking temperature of the world volume black hole geometry is identically zero, $T_{H ; D 5}=0$. We note, however, that the world-volume Hawking temperature $T_{H ; D 5}$ (41) increases monotonically with increasing the angular velocity $\omega$, by which the worldvolume horizon size $r_{H}$ grows. In this case, changing $r_{0}$ in (41) changes the scale, but not the functional form and behavior of the temperature (see Figure 2).

We further notice that by taking into account the backreaction of the above solution to the KW gravity dual, $a d S_{5} \times T^{1,1}$, one accordingly awaits the D5-brane to form a mini black hole in $\mathrm{KW}$, in the probe limit. Thus, the dual gauge theory couples to a thermal object and describes a locally thermal vacuum. In the KW example here, the configuration is dual to $\mathcal{N}=1$ gauge theory coupled to a defect flavor. As the gauge theory itself is at zero temperature while the defect flavor is at finite temperature (41), we conclude that the configuration describes nonequilibrium steady state. However, as we shall show below, the energy from the flavor sector will in the end dissipate to the gauge theory.

So far, in this example, we neglected the backreaction of the D5-brane to the KW gravity dual, as we worked in the probe limit. It is useful to observe the extend this is justifiable. To this end, we derive the components of the energy-stress tensor of our D-brane system. We first note that the energy-stress tensor and its equation of motion take the same form as in (27), implying the conserved energy (28). We then note that for our rotating D5-brane the components of energy-stress tensor in (27) take the form:

$$
\begin{aligned}
\sqrt{-g} J_{t ; D 5}^{t} & \equiv \frac{\tilde{T}_{D 5} r\left(1+r^{2}\left(\phi^{\prime}\right)^{2} / 6\right)}{\sqrt{1+r^{2}\left(\phi^{\prime}\right)^{2} / 6-L^{4} \dot{\phi}^{2} / 6 r^{2}}} \\
& =\frac{\tilde{T}_{D 5}\left(r^{6}-r_{0}^{4} L^{4} \bar{\omega}^{2}\right)}{r^{2} \sqrt{\left(r^{4}-r_{0}^{4}\right)\left(r^{2}-L^{4} \bar{\omega}^{2}\right)}},
\end{aligned}
$$

$$
\begin{aligned}
\sqrt{-g} J_{r ; D 5}^{r} & \equiv-\frac{\tilde{T}_{D 5} r\left(1-L^{4} \dot{\phi}^{2} / 6 r^{2}\right)}{\sqrt{1+r^{2}\left(\phi^{\prime}\right)^{2} / 6-L^{4} \dot{\phi}^{2} / 6 r^{2}}} \\
& =-\tilde{T}_{D 5}\left(r^{2}-L^{4} \bar{\omega}^{2}\right) \sqrt{\frac{r^{4}-r_{0}^{4}}{r^{2}-L^{4} \bar{\omega}^{2}}}
\end{aligned}
$$

$$
\begin{aligned}
\sqrt{-g} J_{t ; D 5}^{r} & \equiv \frac{\tilde{T}_{D 5} r^{3} \dot{\phi} \phi^{\prime}}{\sqrt{1+r^{2}\left(\phi^{\prime}\right)^{2} / 6-L^{4} \dot{\phi}^{2} / 6 r^{2}}} \\
& =\tilde{T}_{D 5} r_{0}^{2} \bar{\omega} .
\end{aligned}
$$


Using (42)-(44), we can derive the total energy and energy flux of the D-brane system. The total energy of the D5-brane in the above configuration is given by:

$$
E_{D 5}=\tilde{T}_{D 5} \int_{r_{0}}^{\infty} \frac{d r\left(r^{6}-r_{0}^{4} L^{4} \bar{\omega}^{2}\right)}{r^{2} \sqrt{\left(r^{4}-r_{0}^{4}\right)\left(r^{2}-L^{4} \bar{\omega}^{2}\right)}} .
$$

Taking the limit $r \longrightarrow r_{0}$, Eq. (42) shows that the energy density of the (defect) flavor brane, the integrand in (45), enlarges dramatically, and blows up exactly at the minimal extension $r=r_{0}$-dual to the IR scale of conformal and chiral flavor symmetry breakdown. We also note that changing $r_{0}$ only shifts the point where the energy density in (45) blows up, but not changes the behavior of the density (see Figure 2). Therefore, we conclude that at the IR scale of symmetries breakdown, $r=r_{0}$, the backreaction of the D5-brane to the gravity dual metric is large and cannot be neglected and forms a localized black hole centered at the IR scale $r=r_{0}$ in the bulk. The size of the black hole ought to increase due to the energy injected into it from the D5-brane continuously. We note that the energy density does not transform as a scalar under coordinate transformations. In order to obtain the energy flux, we compute the time evolution of the total energy by using the components of the energy-stress tensor (42)-(44) and the conserved energy (28), corresponding to our stationary solutions-consistent with global rotational symmetry, as before. We then notice that in the brane configuration of interest here, with $r_{0}>0$ and $\omega>0$, the component (44) is positive definite, and so we derive from (28) the time evolution of the total energy in the form:

$$
\begin{aligned}
\dot{E}_{D 5} & =\frac{d}{d t} \int d r \sqrt{-g} J_{t ; D 5}^{t}=\int d r \partial_{r}\left(\sqrt{-g} J_{t ; D 5}^{r}\right) \\
& =\left.\sqrt{-g} J_{t ; D 5}^{r}\right|_{r=r_{0}} ^{\infty}=\widetilde{T}_{D 5} r_{0}^{2} \bar{\omega}-\widetilde{T}_{D 5} r_{0}^{2} \bar{\omega}=0 .
\end{aligned}
$$

Relation (46) shows that the energy per unit time injected at the UV boundary $r=\infty$ by some external system equals the energy dissipated from the IR into the bulk, despite the total energy is time-independent, as before. This dissipation of energy from the D5-brane to the bulk will create a localized black hole, centered at $r=r_{0}$ in the bulk. We note that when $\omega=0$, relation (33) is trivially satisfied, on contrary with the case $\omega \neq 0$, corresponding to our stationary solutions-consistent with global rotational symmetry. Given this flow of energy from the probe to the bulk, we thereby conclude by duality that the energy from the defect flavor sector will in the end dissipate into the gauge theory conformal and chiral flavor symmetry breakdown. The injection of energy can also be seen clearly in our stationary solution by introducing cutoffs, as before. We can, again, introduce UV and IR cutoffs to the D5brane solution, such that it extends from $r=r_{\mathrm{IR}}=r_{0}$ to $r=$ $r_{\mathrm{UV}} \gg r_{\mathrm{IR}}$. It is clear from (44) that, again, at both $r_{\mathrm{IR}}$ and $r=r_{\mathrm{UV}}$, we have $J_{t}^{r}>0$. This shows the presence of equal energy flux incoming from $r=r_{\mathrm{UV}}$ and outgoing at $r=r_{\mathrm{IR}}$ at which the energy gets not reflected back but its backreaction will nucleate a localized black hole intaking the injected energy, as before.

3.3. Induced Metric and Temperature on U-like Embedded Probe D5-brane Wrapping adS $\times S^{2} \subset a d S_{5} \times T^{1,1}$ and Spinning about $S^{3} \subset T^{1,1}$. As the third example in our study, in this section, we continue with our analysis of the U-like embedded probe D5-brane and consider, instead, the probe wrapping $a d S_{4} \times S^{2}$ in $a d S_{5} \times T^{1,1}$ reviewed in Sec. 2, and turn on, in addition, spin degrees of freedom. Using spherical symmetry, we allow in our setup, the probe to rotate about the $\psi$-direction of the transverse $S^{3}$ with conserved angular momentum. Thus, in our setup, we allow $\psi$ to have, in addition, time-dependence, so that $\dot{\psi}(r, t)=\omega=$ const., with $\omega$ denoting the angular velocity of the probe. This way, we construct rotating solutions. Hence, the world-volume field is given by $\psi(r, t)$, with other directions fixed.

Therefore, we may consider an ansatzes for the D5-brane world volume fields $\theta, \psi(r, t)=\omega t+f(r)$, and $F_{a b}=0$. Using this ansatzes and the metric (5) in terms of (3), it is easy to derive the components of the induced world volume metric on the D5-brane, $g_{a b}^{D 5}$, and compute the determinant, det $g_{a b}^{D 5}$, resulting the DBI action (6) of the form:

$$
S_{D 5} \simeq-T_{D 5} \int d r d t r^{2} \sqrt{1+\frac{r^{2}\left(\psi^{\prime}\right)^{2}}{9}-\frac{L^{4} \dot{\psi}^{2}}{9 r^{2}}} .
$$

Here, we note that by setting $\dot{\psi}=\omega=0$, our action (47) reduces to that of the probe D5-brane action in the BKS model, (14). As in the BKS model reviewed in Sec. 2, restrict brane motion to the $\psi$-direction of the transverse $S^{3}$ sphere and fix other directions constant. Thus, in our setup we let, in addition, the probe rotate about the $S^{3}$. The equation of motion from the action (47) is:

$$
\begin{aligned}
& \frac{\partial}{\partial r}\left[\frac{r^{4} \psi^{\prime}}{\sqrt{1+\left(r^{2}\left(\psi^{\prime}\right)^{2} / 9\right)-L^{4} \dot{\psi}^{2} / 9 r^{2}}}\right] \\
& \quad=\frac{\partial}{\partial t}\left[\frac{L^{4} \dot{\psi}}{\sqrt{1+\left(r^{2}\left(\psi^{\prime}\right)^{2} / 9\right)-L^{4} \dot{\psi}^{2} / 9 r^{2}}}\right] .
\end{aligned}
$$

Take rotating solutions to (48) of the form:

$$
\psi(r, t)=\omega t+f(r), f(r)=r_{0}^{3} \int_{r_{0}}^{r} \frac{d r}{r^{2}} \sqrt{\frac{r^{2}-L^{4} \bar{\omega}^{2}}{r^{6}-r_{0}^{6}}} .
$$

Here, we set $\bar{\omega}=\omega / 3$ and note that when $\omega=0$, our solution (49) integrates to that of probe D5-brane in the BKS model, [65], reviewed in Sec. 2, with the probe wrapping $a d S_{4} \times S^{2}$ in $a d S_{5} \times T^{1,1}$ (see Eq. (15)). The solution (49) is 
parameterized by $\left(\omega, r_{0}\right)$ and describes probe D5-brane motion, with angular velocity $\omega$ about the transverse $S^{3} C$ $T^{1,1}$, starting and ending up at the boundary. The probe descends from the UV boundary at infinity to the minimal extension $r_{0}$ dual to the IR scale where it bends back up the boundary. We also note that inspection of (49) shows that in the limit $r \longrightarrow$ large, the $\omega$-dependent term is subleading, and therefore the behavior of $d f(r) / d r=f_{r}(r)$ does not depend on $\omega$. This shows that in the large radii limit the solution $f(r)$ in (49) gives the world volume field $\psi(r)$ of the BKS model (see Sec. 2 ) with the boundary values $\psi_{ \pm}$in the asymptotic UV limit, $r \longrightarrow \infty$. Nonetheless, we note that in the IR limit, i.e., when $r \longrightarrow$ small, the $\omega$-dependent term becomes large, and therefore the behavior of $f_{r}(r)$ may depend on $\omega$. However, inspection of (49) shows that one can always fix $L$ and regulate $\omega$, so that in the IR, the behavior of $f_{r}(r)$ with $\omega>0$ is comparable to that of with $\omega=0$. This means that certain $\omega>0$ in the small radii limit in the IR the behavior of $f_{r}(r)$ does compare to that of $\psi^{\prime}(r)=d \phi / d r$ in the BKS model (see Sec. 2), with $\psi^{\prime}(r) \longrightarrow \infty$ in the IR limit $r \longrightarrow r_{0}$, consistent with U-like embedding (see Figure 3).

To derive the induced metric on the D5-brane, we put the rotating solution (49) into the background metric (5) and obtain:

$$
\begin{aligned}
d S_{D 5}^{2}= & -\frac{1}{L^{2}}\left(r^{2}-L^{4} \bar{\omega}^{2}\right) d t^{2}+\frac{L^{2}}{r^{2}}\left[\frac{r^{8}-r_{0}^{6} L^{4} \bar{\omega}^{2}}{r^{2}\left(r^{6}-r_{0}^{6}\right)}\right] d r^{2} \\
& +\frac{2 L^{2} r_{0}^{3} \bar{\omega}}{r^{2}} \sqrt{\frac{r^{2}-L^{4} \bar{\omega}^{2}}{r^{6}-r_{0}^{6}}} d r d t+\frac{r^{2}}{L^{2}}\left(d x_{1}^{2}+d x_{2}^{2}\right) .
\end{aligned}
$$

Here, we note that by setting $\omega=0$, our induced world volume metric (50) reduces to that of the BKS model, [65], reviewed in Sec. 2. In this case, for $r_{0}=0$, the induced world volume metric is that of $a d S_{4} \times S^{2}$, and the dual gauge theory describes the conformal and chiral symmetric phase. On the contrary, for $r_{0}>0$, the induced world volume metric has no adS factor and the conformal invariance of the dual gauge theory must be broken in such case.

In order to find the world volume horizon and Hawking temperature, we first eliminate the relevant cross term. To eliminate the relevant cross-term in (50), we consider a coordinate transformation:

$$
\tau=t-L^{4} \bar{\omega} r_{0}^{3} \int \frac{d r}{r^{2} \sqrt{\left(r^{2}-L^{4} \bar{\omega}^{2}\right)\left(r^{6}-r_{0}^{6}\right)}} .
$$

The induced metric on the rotating D5-brane, (50), then takes the form:

$$
d S_{D 5}^{2}=-\frac{1}{L^{2}}\left(r^{2}-L^{4} \bar{\omega}^{2}\right) d \tau^{2}+\frac{L^{2} r^{4}}{r^{6}-r_{0}^{6}} d r^{2}+\frac{r^{2}}{L^{2}}\left(d x_{1}^{2}+d x_{2}^{2}\right)
$$

The induced world volume metric (52) is not given by the black hole geometry with thermal horizon solving $-g_{\tau \tau}=\left.g^{r r}\right|_{r=r_{H}=0}=0$. Inspection of (52) may seem to indicate though that for a very specific, degenerate choice of parameters, $r_{0}=L^{2 / 3} \bar{\omega}$, the induced world volume metric (52) is given by the black hole geometry. But, when the embedding is fixed U-like $\left(r_{0}>0\right)$, this degeneracy conflicts the continuity of the "would be" world-volume thermal horizon: The "would be" world volume thermal horizon, $r_{H}=L^{2} \bar{\omega}$, grows continuously with increasing the angular velocity continuously from zero, $\omega=0$, to positive definite values, $\omega>0$. This means that, in this limit, when $\omega=0$, by degeneracy, the embedding is rather $\mathrm{V}$-like, and only then U-like, when $\omega>0$. Thus, in this example, there is no continuous world volume horizon consistent with the U-like embedding of the probe.

We therefore conclude, in this example, that the induced world volume metric on the U-like embedded probe D5brane wrapping the $a d S_{4} \times S^{2}$ spacetime (in the BKS model) and rotating about the transverse $S^{3} \subset T^{1,1}$ does not describe the world volume black hole geometry with thermal horizon and Hawking temperature of expected features. Thus, by taking into account the backreaction of the rotating D5-brane to the bulk KW gravity dual, the solution cannot describe a mini black hole in KW, in the probe limit. We thus conclude, in this example, that the dual gauge theory cannot couple to a thermal object to form a locally thermal vacuum and therefore there is no nonequilibrium steady-state formation.

Thus far, in this example, we ignored the backreaction of the rotating D5-brane to the KW gravity dual, as we worked in the probe limit. We can go on and derive the energy-stress tensor of the U-like embedded rotating D5-brane and analyse its backreaction and energy dissipation, as in Secs. 3-4. However, from the point of view of nonequilibrium steady-state formation and its transition to thermal equilibrium, in this example, such an analysis would be redundant. Namely, from (52) and the discussion above, it is clear that there is no nonequilibrium steady-state formation, to begin with. In this sense, the system remains in thermal equilibrium and it is immaterial to find whether or not the system will eventually relax to thermal equilibrium, i.e., whether or not the energy of the rotating D5-brane will eventually flow to the bulk KW dual to energy dissipation from the flavor sector into the gauge theory conformal and chiral flavor symmetry breakdown.

\subsection{Induced Metric and Temperature on U-like Embedded} Probe D3-brane Wrapping ad $S_{2} \times S^{2} \subset a d S_{5} \times T^{1,1}$ and Spinning about $S^{3} \subset T^{1,1}$. As the fourth and final example in our study, in this section, we consider another type of probe, namely, the U-like embedded probe D3-brane wrapping ad $S_{2} \times S^{2}$ in $a d S_{5} \times T^{1,1}$ reviewed in Sec. 2, taking into account additional spin degrees of freedom. Using spherical symmetry, we allow in our setup, the probe to rotate about the $\psi$ -direction of the transverse $S^{3}$ with conserved angular momentum. Thus, in our setup, we allow $\psi$ to have, in addition, time-dependence, so that $\dot{\psi}(r, t)=\omega=$ const., with $\omega$ denoting the angular velocity of the probe. This way, we 

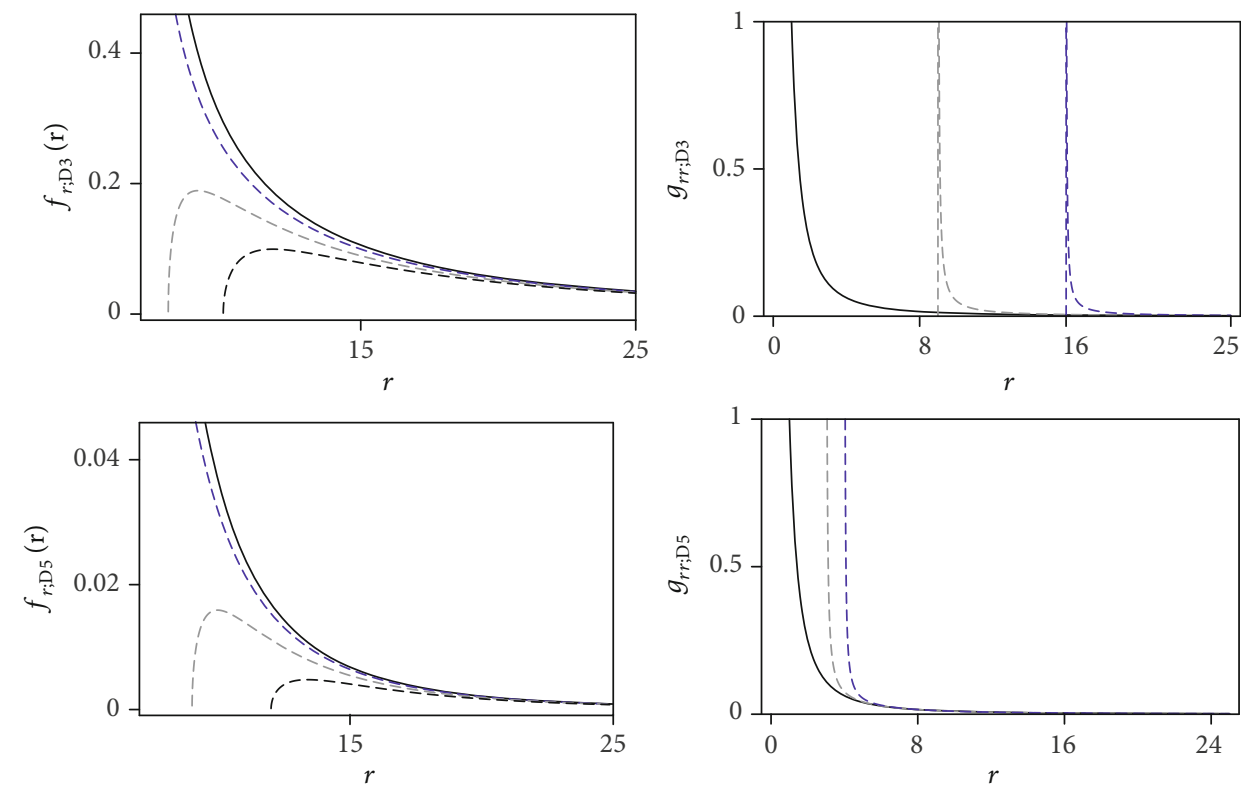

Figure 3: The behavior of the derivative of the D3-brane world volume field with respect to $r$ with $L=1, r_{0}=7, \omega=0$ (black-solid), $\omega=5$ (blue-dashed), $\omega=8$ (gray-dashed), and $\omega=10$ (black-dashed) (up-left). The behavior of the $g_{r r}$ component of the D3-brane induced world volume metric with $L=, r_{0}=0$ (black-solid), $r_{0}=3$ (gray-dashed), and $r_{0}=4$ (blue-dashed) (up-right). The behavior of the derivative of the D5-brane world volume field with respect to $r$ with $L=1, r_{0}=7, \omega=0$ (black-solid), $\omega=5$ (blue-dashed), $\omega=9$ (graydashed), and $\omega=12$ (black-dashed) (down-left). The behavior of the $g_{r r}$ component of the D5-brane induced world volume metric with $L=1, r_{0}=0$ (black-solid), $r_{0}=3$ (gray-dashed), and $r_{0}=4$ (blue-dashed) (down-right).

construct rotating solutions. Hence, the world-volume field is given by $\psi(r, t)$, with other directions fixed.

Thus, we consider an ansatzes for the D3-brane world volume $\psi(r, t)=\omega t+f(r)$, with other directions fixed, and $F_{a b}=0$. Using this ansatzes and the metric (5) in terms of (3), it is easy to derive the components of the induced world volume metric on the D3-brane, $g_{a b}^{D 3}$, and compute the determinant, det $g_{a b}^{D 3}$, resulting the DBI action (6) of the form:

$$
S_{D 3} \simeq-T_{D 3} \int d r d t \sqrt{1+\frac{r^{2}\left(\psi^{\prime}\right)^{2}}{9}-\frac{L^{4} \dot{\psi}^{2}}{9 r^{2}}}
$$

Here, we note that by setting $\dot{\psi}=\omega=0$, our action (53) reduces to that of the probe D3-brane action in the BKS model, (8). As in the BKS model reviewed in Sec. 2, restrict brane motion to the $\psi$-direction of the transverse $S^{3}$ sphere and fix other directions constant. Thus, in our set up, we let, in addition, the probe rotate about the $S^{3}$. The equation of motion from the action (53) is:

$$
\begin{gathered}
\frac{\partial}{\partial r}\left[\frac{r^{2} \psi^{\prime}}{\sqrt{1+\left(r^{2}\left(\psi^{\prime}\right)^{2} / 9\right)-L^{4} \dot{\psi}^{2} / 9 r^{2}}}\right] \\
\quad=\frac{\partial}{\partial t}\left[\frac{L^{4} \dot{\psi}}{r^{2} \sqrt{1+\left(r^{2}\left(\psi^{\prime}\right)^{2} / 9\right)-L^{4} \dot{\psi}^{2} / 9 r^{2}}}\right] .
\end{gathered}
$$

Take rotating solutions to (54) of the form:

$$
\psi(r, t)=\omega t+f(r), f(r)=3 r_{0} \int_{r_{0}}^{r} \frac{d r}{r^{2}} \sqrt{\frac{r^{2}-L^{4} \bar{\omega}^{2}}{r^{2}-r_{0}^{2}}}
$$

Here, we set $\bar{\omega}=\omega / 3$ and note that when $\omega=0$, our solution (55) integrates to that of probe D3-brane in the BKS model, [65], reviewed in Sec. 2, with the probe wrapping ad $S_{2} \times S^{2}$ in $a d S_{5} \times T^{1,1}$ (see Eq. (18)). The solution (55) is parameterized by $\left(\omega, r_{0}\right)$ and describes probe D3-brane motion, with angular velocity $\omega$ about the transverse $S^{3} \mathrm{C}$ $T^{1,1}$, starting and ending up at the boundary. The probe descends from the UV boundary at infinity to the minimal extension $r_{0}$ dual to the IR scale where it bends back up the boundary. We also note that inspection of (55) shows that in the limit $r \longrightarrow$ large, the $\omega$-dependent term becomes subleading, and therefore the behavior of $d f(r) / d r=f_{r}(r)$ does not depend on $\omega$. This shows that in the large radii limit the solution $f(r)$ in (55) gives the world volume field $\psi(r)$ of the BKS model (see Sec. 2) with the boundary values $\psi_{ \pm}$ in the asymptotic UV limit, $r \longrightarrow \infty$. Nonetheless, we note that in the IR limit, i.e., when $r \longrightarrow$ small, the $\omega$-dependent term becomes large, and therefore the behavior of $f_{r}(r)$ may depend on $\omega$. However, inspection of (55) shows that one can always choose $\omega>0$ so that in the IR the behavior of $f_{r}(r)$ with $\omega>0$ is comparable to that of with $\omega=0$. This means that for certain $\omega>0$ in the small radii limit in the IR the behavior of $f_{r}(r)$ does compare to that of $\psi^{\prime}(r)=d \phi / d r$ in the BKS model (see Sec. 2), 
with $\psi^{\prime}(r) \longrightarrow \infty$ in the IR limit $r \longrightarrow r_{0}$, consistent with U-like embedding (see Figure 3).

To derive the induced metric on the D3-brane, we put the rotating solution (55) into the background metric (5) and obtain:

$$
\begin{aligned}
d S_{D 3}^{2}= & -\frac{1}{L^{2}}\left(r^{2}-L^{4} \bar{\omega}^{2}\right) d t^{2}+\frac{L^{2}}{r^{2}}\left[\frac{r^{4}-r_{0}^{2} L^{4} \bar{\omega}^{2}}{r^{2}\left(r^{2}-r_{0}^{2}\right)}\right] d r^{2} \\
& +\frac{2 L^{2} r_{0} \bar{\omega}}{r^{2}} \sqrt{\frac{r^{2}-L^{4} \bar{\omega}^{2}}{r^{2}-r_{0}^{2}}} d r d t .
\end{aligned}
$$

To eliminate the cross-term in (56), we consider a coordinate transformation:

$$
\tau=t-L^{4} \bar{\omega} r_{0} \int \frac{d r}{r^{2} \sqrt{\left(r^{2}-L^{4} \bar{\omega}^{2}\right)\left(r^{2}-r_{0}^{2}\right)}} .
$$

The induced world volume metric on the rotating probe D3-brane then reads:

$$
d S_{D 3}^{2}=-\frac{1}{L^{2}}\left(r^{2}-L^{4} \bar{\omega}^{2}\right) d \tau^{2}+\frac{L^{2}}{r^{2}-r_{0}^{2}} d r^{2} .
$$

The induced world volume metric (58) is not given by the black hole geometry with thermal horizon solving $-g_{\tau \tau}=\left.g^{r r}\right|_{r=r_{H}}=0$. Inspection of (58) may seem to indicate though that for a very specific, degenerate choice of parameters, $r_{0}=L^{2} \bar{\omega}$, the induced world volume metric (58) is that of the BTZ black hole metric minus the angular coordinate. But, when the embedding is fixed U-like $\left(r_{0}>0\right)$, this degeneracy is inconsistent with the continuity of the "would be" world volume horizon: The "would be" world volume horizon, $r_{H}=L^{2} \bar{\omega}$, has to grow continuously with increasing the angular velocity continuously, i.e., from zero, $\omega=0$, to any positive definite values, $\omega>0$. This implies that when $\omega=0$, the embedding is V-like, and only then U-like, when $\omega>0$. Thus, in this example, there is no continuous world volume horizon consistent with the U-like embedding of the probe.

We therefore conclude, in this example, that the induced world volume metric on the U-like embedded probe D3brane wrapping the $a d S_{2} \times S^{2}$ spacetime (in the BKS model) and rotating about the transverse $S^{3} \subset T^{1,1}$ does not describe the world volume black hole geometry with thermal horizon and Hawking temperature of expected features. Thus by taking into account the backreaction of the rotating D3brane to the bulk KW gravity dual, the solution cannot describe a mini black hole in $\mathrm{KW}$, in the probe limit. We thus conclude, in this example, that the dual gauge theory cannot couple to a thermal object to form a locally thermal vacuum, and therefore there is no nonequilibrium steady-state formation. This is in contrast with the conformal $a d S_{5} \times S^{5}$ solution where the induced world volume metric on the rotating probe D3-brane describes the world volume black hole geometry with thermal horizon, $r_{H}=\omega$, and Hawking temperature, $T_{H}=\sqrt{3} \omega / 2 \pi$, of expected features [34].).

Thus far, in this example, we ignored the backreaction of the rotating D3-brane to the KW gravity dual, as we worked in the probe limit. We can go on and derive the energy-stress tensor of the U-like embedded rotating D3-brane and analyse its backreaction and energy dissipation, as in Secs. 3-4. However, from the point of view of nonequilibrium steady state formation and its transition to thermal equilibrium, in this example, such an analysis would be redundant. Namely, from (58) and the discussion above, it is clear that there is no nonequilibrium steady-state formation, to begin with. In this sense, the system remains in thermal equilibrium and it is immaterial to find whether or not the system will eventually relax to thermal equilibrium, i.e., whether or not the energy of the rotating D3-brane will eventually flow to the bulk KW dual to energy dissipation from the flavor sector into the gauge theory conformal and chiral flavor symmetry breakdown.

\section{Discussion}

In this work, we studied the induced world volume metrics of all U-like embedded type IIB rotating probe flavor Dp-branes $(p=3,5,7)$ in the zero temperature KW gravity dual of gauge field theories with spontaneous breakdown of the conformal and chiral flavor symmetry. Furthermore, we studied the related energy-stress tensors and energy flow of the U-like embedded rotating probes. By gauge/gravity duality, the induced Hawking temperatures of such rotating probes in gravity duals correspond to the temperatures of flavors at finite R-charge and spontaneous breakdown of the conformal and chiral flavor symmetry, and the energy flow from such probes to the bulk to the energy dissipation from the flavor sectors to the gauge theory conformal and chiral flavor symmetry breakdown. Such nonequilibrium finite-spin systems and their energy flow have been studied in previous work by using the Kuperstein-Sonnenschein holographic model. The model consisted of adding spin to U-like embedded spacetime filling probe flavor D7-branes in the KW gravity dual of CFT with spontaneous conformal and chiral flavor symmetry breakdown. The aim of this work was to generalize and complete such previous analyses by considering new Ulike embeddings dual to dCFTs with spontaneous breakdown of the conformal and chiral flavor symmetry. This involved studying the induced world volume metrics of the remaining type IIB probe flavor branes in the more generic BKS holographic models with additional spin. The models consisted of adding spin to U-like embedded probe flavor D3-, and D5-branes localized in part in the KW gravity dual of dCFTs with conformal and chiral flavor symmetry breakdown. The motivation of this work was to determine if such new dCFT embeddings could reveal new features of thermalization and include novel examples of nonequilibrium systems. The motivation was thus to construct and classify all such novel examples of nonequilibrium systems in holographic type IIB models dual to (d)CFTs with spontaneous breakdown of the conformal and chiral flavor symmetry. 
We started our analysis by considering different examples of U-like embedded type IIB rotating probe branes wrapping the same three-cycle in the KW gravity dual of (d)CFT with (defect) flavors at finite R-charge and spontaneous breakdown of the conformal and chiral flavor symmetry. We first reviewed the main aspects of our previous casestudy of U-like embedded rotating probe D7-branes in the KW gravity dual of CFT with spacetime filling flavors at finite $\mathrm{R}$-charge and spontaneous breakdown of the conformal and chiral flavor symmetry. We then continued our analysis by considering, as our first novel example, the U-like embedded rotating probe D5-brane in the KW gravity dual of dCFT with codimension two defect flavors at finite R-charge and spontaneous breakdown of the conformal and chiral flavor symmetry. We computed the induced world volume metric of the probe D5-brane and found, in this case, the world volume black hole solution and energy dissipation similar to that of the probe D7-brane. We found that when additional spin is turned on, the induced world volume metric on the U-like embedded probe D5-brane admits a thermal horizon and Hawking temperature of expected features. By gauge/gravity duality, we thus found that when the codimension two defect flavor sector of the dCFT with spontaneous conformal and chiral flavor symmetry breakdown admits finite R-charged, it becomes thermal. We then noted that by taking into account the backreaction of the brane solution to the bulk, one naturally expects the U-like embedded rotating probe to form a mini black hole in the bulk KW gravity dual of dCFT with spontaneous breakdown of the conformal and chiral flavor symmetry, in the probe limit. Thus, we found the U-like embedded rotating probe describing a thermal object and hence describing a locally thermal vacuum in the dual dCFT with spontaneous conformal and chiral symmetry breakdown. In such example, the system was dual to $\mathcal{N}=1$ conformal gauge field theory coupled to a localized flavored quark. Since the dCFT itself was at zero temperature while its codimension two flavored quark sector was at finite temperature, we found in this example that such a system describes nonequilibrium steady state in the dCFT with conformal and chiral flavor symmetry breakdown. However, by computing the energy-stress tensor of the probe, we found that the energy from the probes will eventually flow to the bulk KW and form, with the large backreaction in the IR, a localized black hole in the bulk. By gauge/gravity duality, we thus find that the energy from the defect flavor sector will eventually dissipate into the $\mathcal{N}=1$ gauge theory conformal and chiral flavor symmetry breakdown. The similarities between the induced metrics of the probe D5-brane, in this example, and of the D7-brane are no surprise, after all, both probes wrap the same three-cycle. Nonetheless, these embeddings are dual to different field theories including CFT and dCFT with spontaneous flavor symmetry breakdown. To this end, we may also note that such embeddings dual to different nonequilibrium systems may simply be unstable and require perturbative analysis. But, by energy dissipation, such systems will eventually relax to equilibrium, as discussed above.

We then further continued and completed our analysis by considering further novel examples of U-like embedded type IIB rotating probe branes wrapping the same two-cycle in the KW gravity dual of dCFT with defect flavors at finite R-charge and spontaneous breakdown of the conformal and chiral flavor symmetry. We considered, as our second and third novel examples, the alternative U-like embedded rotating probe $\mathrm{D} 5$-brane and the $\mathrm{U}$-like embedded rotating probe D3-brane in the KW gravity dual of dCFT with codimension one or three defect flavors at finite R-charge and spontaneous breakdown of the conformal and chiral flavor symmetry. We computed the induced world volume metrics on these probes and found their behavior the same. We found in these examples, that when additional spin is turned on, the induced world volume metrics on the U-like embedded probe D5- and D3-branes do not describe the black hole geometry with thermal horizons and Hawking temperatures of expected features. We first noted, in both cases, that in order to render the induced world volume metrics of the probes of the form akin to the black hole geometry, the parameter space has to be degenerate, i.e., the minimal extension-dual to the IR scale of symmetry breakdown has to equal the angular velocity-dual to R-charge, $r_{0}=\omega$. However, we then found, in these examples, that when the embedding is U-like, $r_{0}>0$, the angular velocity, $\omega$, cannot increase continuously, as it should-from zero to positive definite values. We thus found that the degenerate choice of parameters obstructs, in turn, the continuous growth of the "would be" world volume horizons. We therefore found, in these examples, that the induced world volume metrics on the rotating probes do not describe the black hole geometry with thermal horizon and Hawking temperature of expected features. By gauge/gravity duality, we thus found, in these examples, that when the codimension one or three defect flavor sectors of the dCFT with spontaneous conformal and chiral flavor symmetry breakdown become R-charged, they cannot become thermal. We thus exclude these embeddings as possible examples of dCFTs describing flavor thermalization and nonequilibrium steady-state formation. The similarities between the induced worldvolume metrics of the probe D5brane and of the D3-brane in these examples are no surprise, after all, both probes wrap the same two-cycle. Nonetheless, these embeddings are dual to different field theories including different dCFTs.

We conclude from our new embeddings the novel feature that embedding the probes U-like obstructs the induced world volume metrics on rotating probes to describe the black hole geometry. We conclude that the obstruction is subject to the world volume and topology of the nontrivial internal cycle wrapped by the probe. We thus conclude that in U-like embeddings, the induced world volume black hole solutions are classified according to the topologically nontrivial embeddings of the probes. We conclude by duality the novel feature that in field theories with the R-charged flavor sector, spontaneous conformal and chiral flavor symmetry breakdown obstructs flavor thermalization and nonequilibrium state formation. We conclude that the obstruction is subject to the type of flavor and (co)dimension of dual spacetime where the flavor fields reside. We thus conclude that in field theories with spontaneous conformal and chiral flavor symmetry breakdown, nonequilibrium systems are classified according to the kind of field theory and type 
of flavor inserted into the theory. We conclude, however, that such systems will eventually relax to equilibrium.

There are the following limitations to our work. In the examples we constructed, we were unable to fully solve the $D p$-brane $(p=3,5,7)$ equations of motion to determine the explicit analytic form of the $D p$-brane world volume scalar fields, when conserved angular motion was turned on. Therefore, in our examples, we were not able to provide full details about the probe $D p$-brane solution itself, when the angular velocity was set nonvanishing. Nevertheless, in order to derive the induced world volume metrics and compute the induced world volume Hawking temperatures, we did not need to find the explicit form of the $D p$-brane world volume scalar fields. However, in all examples we constructed, we deliberately chose ansätze of solutions and induced world volume metrics that did reproduce those of the BKS models, when we turned off the angular velocity. Moreover, in all examples, we noted that independent from the value of angular velocity, in the large radii limit, the radial derivatives of the world volume field in our ansätze coincide with that of vanishing angular velocity. Thus, in all examples, in the large radii limit, our ansätze solved to the $D p$-brane scalar fields with asymptotic UV boundary values of the BKS models. Also, in all our examples we noted that in the small radii limit, in the IR, for specific range of angular velocities, the radial derivatives of the world volume field in our ansätze behave as that of the BKS models, consistent with U-like embeddings. Furthermore, in our analysis, we refrained from turning on worldvolume gauge fields dual to nonvanishing external fields in the gauge theory. This is chiefly because we did not need to do so, in order to determine if the induced world volume metrics on rotating probes in BKS models admit thermal horizons and Hawking temperatures of expected features. In other words, in the dual picture, this means that in order to determine if gauge theories with spontaneous conformal and chiral flavor symmetry breakdown admit flavor thermalization and nonequilibrium formation, we did not turn on external fields. However, it would be interesting to study the phenomenological implications of nonvanishing external fields in such models. For instance, in well known conformal models in the literature, it has been shown that by turning on a worldvolume gauge field $A_{1}=-E t+h(r)$ with electric $E=-F_{01}$ and magnetic $F_{r 1}$ fields (in place of turning on rotation $-\phi=\omega t+f(r)$ ), the induced metrics of probes describe thermal horizons and Hawking temperatures different from those of the background. However, we note that such an ansatzes compares to the ansatzes for the worldvolume scalar describing rotation. We also note that the related field equation and induced worldvolume black hole solution of such model compare to those of the conformal model with worldvolume scalar describing rotation. We thus infer from these similarities, it is very unlikely that our main conclusions change if turning on the worldvolume gauge field on U-like embedded probes.

The other crucial aspect of the BKS models that we did not analyse in this paper is the perturbative expansion and identification of the dilaton-the Goldstone boson of conformal symmetry breakdown. It is certainly a curious question of how to extend the perturbative expansion and describe the Goldstone boson of the system at finite R-charge. Confining ourselves to the most important example of spacetime filling flavors, we may then first pay attention to the following basic facts from the pure spectrum model. The perturbative expansion appropriate for the model to determine the Goldstone boson of the system involves deforming the world volume embedding ansatzes through a transformation from "polar" to "Cartesian" coordinates. Within a definite set of such coordinates, the model computes the induced metric and action for fluctuation fields yielding the Schrdinger-like equation of motion. The model then adopts certain symmetries and boundary conditions and solves the equation by numerical method and determines the spectrum revealing the Goldstone boson of broken conformal symmetry. In line with this, we then note how such construction may be realized for our case-study of spacetime filling flavors. For instance, we may aim to modify the perturbative expansion of the pure model by including additional spin degrees of freedom and so determine the Goldstone boson of the system in the presence of additional rotation-dual to finite Rcharge. The related Schrdinger-type equation of motion should then include additional angular momenta in the potential and solve to the spectrum revealing the Goldstone boson at finite R-charge. However, we note that field equations with rotational spherical symmetry dual to finite Rcharge are generally and systematically solved in polar coordinate systems, and solving them instead in Cartesian systems is a formidable task. Moreover, in the attempt to modify the world volume embedding ansatzes of the pure spectrum model to include rotation, we found such configurations ambiguous, to begin with. Namely, we were unable to derive, in the first place, the induced world volume metric in the form given by the rotating black hole geometry dual to flavor thermalization at finite R-charge in the nonconformal gauge theory. We thus found such configurations rather unwieldy to identify the Goldstone boson, when the system is at finite R-charge. We suspect though that this issue may be technically more involved and therefore requires further investigation, perhaps by alternative methods, which go beyond the scope of this paper. The analysis of this work may be extended in two obvious ways. One obvious extension is to study probe flavor brane thermalization in U-like embeddings in more generic type IIB holographic models, such as the regular KS and singular KT gravity duals of $\mathcal{N}=1$ nonconformal gauge theories including confinement and chiral symmetry breaking, and RG flow, respectively. The other obvious extension is to study probe flavor brane thermalization in U-like embeddings of type IIA holographic BKS models based on ABJM theory. We leave these extensions for our future studies.

\section{Data Availability}

This manuscript contains all data used for this study.

\section{Conflicts of Interest}

The authors declare that they have no conflicts of interest. 


\section{Acknowledgments}

I am very grateful to the editor and, especially, to the reviewers for useful comments on the paper.

\section{References}

[1] J. Maldacena, "The large N limit of superconformal field theories and supergravity," International Journal of Theoretical Physics, vol. 38, no. 4, pp. 1113-1133, 1999.

[2] S. S. Gubser, I. R. Klebanov, and A. M. Polyakov, "Gauge theory correlators from non-critical string theory," Physics Letters $B$, vol. 428, no. 1-2, pp. 105-114, 1998.

[3] E. Witten, "Anti de Sitter space and holography," Advances in Theoretical and Mathematical Physics, vol. 2, no. 2, pp. 253291, 1998.

[4] M. Ammon and J. Erdmenger, Gauge/gravity duality: foundations and applications, Cambridge University Press, Cambridge, 2015.

[5] H. Nastase, Introduction to the ADS/CFT Correspondence, Cambridge University Press, Cambridge, 2015.

[6] A. Karch and E. Katz, "Adding flavor to AdS/CFT," Journal of High Energy Physics, vol. 2002, no. 6, p. 043, 2002.

[7] A. Karch and L. Randall, "Localized gravity in string theory," Physical Review Letters, vol. 87, no. 6, article 061601, 2001.

[8] A. Karch and L. Randall, "Open and closed string interpretation of SUSY CFT's on branes with boundaries," Journal of High Energy Physics, vol. 2001, no. 6, p. 063, 2001.

[9] M. Kruczenski, D. Mateos, R. C. Myers, and D. J. Winters, "Meson spectroscopy in AdS/CFT with flavour," Journal of High Energy Physics, vol. 2003, no. 7, p. 049, 2003.

[10] D. Mateos, R. C. Myers, and R. M. Thomson, "Holographic phase transitions with fundamental matter," Physical Review Letters, vol. 97, no. 9, article 091601, 2006.

[11] D. Mateos, R. C. Myers, and R. M. Thomson, "Holographic viscosity of fundamental matter," Physical Review Letters, vol. 98, no. 10, article 101601, 2007.

[12] S. Kobayashi, D. Mateos, S. Matsuura, R. C. Myers, and R. M. Thomson, "Holographic phase transitions at finite baryon density," Journal of High Energy Physics, vol. 2007, no. 2, p. 016, 2007.

[13] D. Mateos, S. Matsuura, R. C. Myers, and R. M. Thomson, "Holographic phase transitions at finite chemical potential," Journal of High Energy Physics, vol. 2007, no. 11, p. 085, 2007.

[14] J. Babington, J. Erdmenger, N. Evans, Z. Guralnik, and I. Kirsch, "Chiral symmetry breaking and pions in nonsupersymmetric gauge/gravity duals," Physical Review D, vol. 69, no. 6, article 066007, 2004.

[15] R. Apreda, J. Erdmenger, N. Evans, and Z. Guralnik, "Strong coupling effective Higgs potential and a first order thermal phase transition from AdS/CFT duality," Physical Review D, vol. 71, no. 12, article 126002, 2005.

[16] G. Itsios, N. Jokela, and A. V. Ramallo, "Collective excitations of massive flavor branes," Nuclear Physics B, vol. 909, pp. 677724, 2016.

[17] A. Karch and A. O'Bannon, "Metallic AdS/CFT," Journal of High Energy Physics, vol. 2007, no. 9, p. 024, 2007.

[18] M. Ammon, J. Erdmenger, M. Kaminski, and P. Kerner, "Superconductivity from gauge/gravity duality with flavor," Physics Letters B, vol. 680, no. 5, pp. 516-520, 2009.
[19] M. Ammon, J. Erdmenger, P. Kerner, and M. Kaminski, "Flavor superconductivity from gauge/gravity duality," Journal of High Energy Physics, vol. 2009, no. 10, p. 067, 2009.

[20] N. Evans, A. Gebauer, K. Y. Kim, and M. Magou, "Holographic description of the phase diagram of a chiral symmetry breaking gauge theory," Journal of High Energy Physics, vol. 2010, no. 3, p. 132, 2010.

[21] K. Jensen, A. Karch, and E. G. Thompson, "A holographic quantum critical point at finite magnetic field and finite density," Journal of High Energy Physics, vol. 2010, no. 5, p. 015, 2010.

[22] K. Jensen, A. Karch, D. T. Son, and E. G. Thompson, "Holographic Berezinskii-Kosterlitz-Thouless Transitions," Physical Review Letters, vol. 105, no. 4, article 041601, 2010.

[23] N. Evans, A. Gebauer, K. Y. Kim, and M. Magou, "Phase diagram of the D3/D5 system in a magnetic field and a BKT transition," Physics Letters B, vol. 698, no. 1, pp. 91-95, 2011.

[24] S. A. Hartnoll, J. Polchinski, E. Silverstein, and D. Tong, "Towards strange metallic holography," Journal of High Energy Physics, vol. 2010, no. 4, p. 120, 2010.

[25] C. P. Herzog, "Lectures on holographic superfluidity and superconductivity," Journal of Physics A, vol. 42, no. 34, article 343001, 2009.

[26] S. A. Hartnoll, "Lectures on holographic methods for condensed matter physics," Classical and Quantum Gravity, vol. 26, no. 22, article 224002, 2009.

[27] T. Faulkner, N. Iqbal, H. Liu, J. McGreevy, and D. Vegh, "From black holes to strange metals," https://arxiv.org/abs/1003.1728.

[28] J. McGreevy, "Holographic duality with a view toward manybody physics," Advances in High Energy Physics, vol. 2010, Article ID 723105, 54 pages, 2010.

[29] H. Liu, J. McGreevy, and D. Vegh, "Non-Fermi liquids from holography," Physical Review D, vol. 83, no. 6, article 065029, 2011.

[30] T. Faulkner, H. Liu, J. McGreevy, and D. Vegh, "Emergent quantum criticality, Fermi surfaces, and $\mathrm{AdS}_{2}$," Physical Review D, vol. 83, no. 12, article 125002, 2011.

[31] A. O'Bannon, "Toward a holographic model of superconducting fermions," Journal of High Energy Physics, vol. 2009, no. 1, p. 074, 2009.

[32] S. P. Kumar, "Spinning flavor branes and fermion pairing instabilities," Physical Review D, vol. 84, no. 2, article 026003, 2011.

[33] N. Evans and E. Threlfall, "Chemical potential in the gravity dual of a $2+1$ dimensional system," Physical Review D, vol. 79, no. 6, article 066008, 2009.

[34] S. R. Das, T. Nishioka, and T. Takayanagi, "Probe branes, time-dependent couplings and thermalization in AdS/CFT," Journal of High Energy Physics, vol. 2010, no. 7, p. 71, 2010.

[35] H. Ebrahim and M. Ali-Akbari, "Dynamically probing strongly-coupled field theories with critical point," Physics Letters $B$, vol. 783, pp. 43-50, 2018.

[36] L. Shahkarami, H. Ebrahim, M. Ali-Akbari, and F. Charmchi, "Far-from-equilibrium initial conditions probed by a nonlocal observable," Physics Letters B, vol. 773, pp. 91-97, 2017.

[37] A. Hajilou, M. Ali-Akbari, and F. Charmchi, "A classical string in Lifshitz-Vaidya geometry," European Physical Journal C, vol. 78, no. 5, p. 424, 2018.

[38] S. F. Taghavi and A. Vahedi, "Equilibrium instability of chiral mesons in external electromagnetic field via AdS/CFT," Journal of High Energy Physics, vol. 2016, no. 6, p. 053, 2016. 
[39] M. Ali-Akbari and S. F. Taghavi, "Chiral magnetic effect in the anisotropic quark-gluon plasma," Journal of High Energy Physics, vol. 2015, no. 4, p. 181, 2015.

[40] M. Ali-Akbari, H. Ebrahim, and Z. Rezaei, "Probe branes thermalization in external electric and magnetic fields," Nuclear Physics B, vol. 878, pp. 150-168, 2014.

[41] M. Ali-Akbari and H. Ebrahim, "Thermalization in external magnetic field," Journal of High Energy Physics, vol. 2013, no. 3, p. 045, 2013.

[42] S. Nakamura and H. Ooguri, "Out of equilibrium temperature from holography," Physical Review D, vol. 88, no. 12, article 126003, 2013.

[43] M. Ali-Akbari and H. Ebrahim, "Meson thermalization in various dimensions," Journal of High Energy Physics, vol. 2012, no. 4, p. 145, 2012.

[44] M. Ali-Akbari and U. Gursoy, "Rotating strings and energy loss in non-conformal holography," Journal of High Energy Physics, vol. 2012, no. 1, p. 105, 2012.

[45] S. R. Das, "Holographic quantum quench," Journal of Physics: Conference Series, vol. 343, article 012027, 2012.

[46] C. Hoyos, T. Nishioka, and A. O’Bannon, “A chiral magnetic effect from AdS/CFT with flavor," Journal of High Energy Physics, vol. 2011, no. 10, p. 084, 2011.

[47] J. G. Russo and P. K. Townsend, "Accelerating branes and brane temperature," Classical and Quantum Gravity, vol. 25, no. 17, article 175017, 2008.

[48] M. Chernicoff and A. Guijosa, "Acceleration, energy loss and screening in strongly-coupled gauge theories," Journal of High Energy Physics, vol. 2008, no. 6, p. 005, 2008.

[49] A. Paredes, K. Peeters, and M. Zamaklar, "Temperature versus acceleration: the Unruh effect for holographic models," Journal of High Energy Physics, vol. 2009, no. 4, p. 015, 2009.

[50] C. Athanasiou, P. M. Chesler, H. Liu, D. Nickel, and K. Rajagopal, "Synchrotron radiation in strongly coupled conformal field theories," Physical Review D, vol. 81, no. 12, article 126001, 2010.

[51] E. Caceres, M. Chernicoff, A. Guijosa, and J. F. Pedraza, "Quantum fluctuations and the Unruh effect in stronglycoupled conformal field theories," Journal of High Energy Physics, vol. 2010, no. 6, p. 078, 2010.

[52] T. Hirata, T. Takayanagi, and S. Mukohyama, "Decaying Dbranes and moving mirrors," Journal of High Energy Physics, vol. 2008, no. 5, p. 089, 2008.

[53] T. Hirayama, P. W. Kao, S. Kawamoto, and F. L. Lin, "Unruh effect and holography," Nuclear Physics B, vol. 844, no. 1, pp. 1-25, 2011.

[54] D. Kaviani and A. E. Mosaffa, "Temperature in the throat," Nuclear Physics B, vol. 910, pp. 724-753, 2016.

[55] D. Kaviani, "D7-brane dynamics and thermalization in the Kuperstein-Sonnenschein model," Nuclear Physics B, vol. 919, pp. 142-172, 2017.

[56] I. R. Klebanov and M. J. Strassler, "Supergravity and a confining gauge theory: duality cascades and $\chi$ SB-resolution of naked singularities," Journal of High Energy Physics, vol. 2000, no. 8, p. 052, 2000.

[57] I. R. Klebanov and A. A. Tseytlin, "Gravity duals of supersymmetric $\mathrm{SU}(\mathrm{N}) \times \mathrm{SU}(\mathrm{N}+\mathrm{M})$ gauge theories," Nuclear Physics $B$, vol. 578, no. 1-2, pp. 123-138, 2000.

[58] I. R. Klebanov and E. Witten, "Superconformal field theory on threebranes at a Calabi-Yau singularity," Nuclear Physics B, vol. 536, no. 1-2, pp. 199-218, 1998.
[59] A. Buchel, "Finite temperature resolution of the KlebanovTseytlin singularity," Nuclear Physics B, vol. 600, no. 2, pp. 219-234, 2001.

[60] A. Buchel, I. R. Klebanov, C. P. Herzog, L. P. Zayas, and A. A. Tseytlin, "Nonextremal gravity duals for fractional D-3 branes on the conifold," Journal of High Energy Physics, vol. 2001, no. 4, p. 033, 2001.

[61] S. S. Gubser, C. P. Herzog, I. R. Klebanov, and A. A. Tseytlin, "Restoration of chiral symmetry: a supergravity perspective," Journal of High Energy Physics, vol. 2001, no. 5, p. 028, 2001.

[62] O. Aharony, A. Buchel, and P. Kerner, "Black hole in the throat: thermodynamics of strongly coupled cascading gauge theories," Physical Review D, vol. 76, no. 8, article 086005, 2007.

[63] A. Buchel, "Klebanov-Strassler black hole," Journal of High Energy Physics, vol. 2019, no. 1, p. 207, 2019.

[64] P. Candelas and X. C. de la Ossa, "Comments on conifolds," Nuclear Physics B, vol. 342, no. 1, pp. 246-268, 1990.

[65] O. Ben-Ami, S. Kuperstein, and J. Sonnenschein, "On spontaneous breaking of conformal symmetry by probe flavour Dbranes," Journal of High Energy Physics, vol. 2014, no. 3, p. 45, 2014.

[66] V. G. Filev, M. Ihl, and D. Zoakos, "Holographic bilayer/monolayer phase transitions," Journal of High Energy Physics, vol. 2014, no. 7, 2014.

[67] V. G. Filev, M. Ihl, and D. Zoakos, "A novel (2+1)-dimensional model of chiral symmetry breaking," Journal of High Energy Physics, vol. 2013, no. 12, p. 072, 2013.

[68] S. Kuperstein and J. Sonnenschein, "A new holographic model of chiral symmetry breaking," Journal of High Energy Physics, vol. 2008, no. 9, 2008.

[69] M. Ihl, A. Kundu, and S. Kundu, "Back-reaction of nonsupersymmetric probes: phase transition and stability," Journal of High Energy Physics, vol. 2012, no. 12, p. 070, 2012.

[70] M. S. Alam, V. S. Kaplunovsky, and A. Kundu, "Chiral symmetry breaking and external fields in the KupersteinSonnenschein model," Journal of High Energy Physics, vol. 2012, no. 4, p. 111, 2012.

[71] T. Sakai and J. Sonnenschein, "Probing flavored mesons of confining gauge theories by supergravity," Journal of High Energy Physics, vol. 2003, no. 9, p. 047, 2003.

[72] A. Dymarsky, S. Kuperstein, and J. Sonnenschein, "Chiral symmetry breaking with non-SUSY D7-branes in ISD backgrounds," Journal of High Energy Physics, vol. 2009, no. 8, p. 005, 2009.

[73] T. Sakai and S. Sugimoto, "Low energy hadron physics in holographic QCD," Progress of Theoretical Physics, vol. 113, no. 4, pp. 843-882, 2005.

[74] T. Sakai and S. Sugimoto, "More on a holographic dual of QCD," Progress in Theoretical Physics, vol. 114, no. 5, pp. 1083-1118, 2005.

[75] P. Ouyang, "Holomorphic D7-branes and flavored N=1 gauge theories," Nuclear Physics B, vol. 699, no. 1-2, pp. 207-225, 2004.

[76] T. S. Levi and P. Ouyang, "Mesons and flavor on the conifold," Physical Review D, vol. 76, no. 10, article 105022, 2007.

[77] S. Kuperstein, "Meson spectroscopy from holomorphic probes on the warped deformed conifold," Journal of High Energy Physics, vol. 2005, no. 3, p. 014, 2005.

[78] F. Benini, S. Cremonesi, F. Canoura, A. V. Ramallo, and C. Núnez, "Unquenched flavors in the Klebanov-Witten 
model," Journal of High Energy Physics, vol. 2007, no. 2, p. 090, 2007.

[79] F. Benini, S. Cremonesi, F. Canoura, A. V. Ramallo, and C. Núñez, "Backreacting flavors in the Klebanov-Strassler background," Journal of High Energy Physics, vol. 2007, no. 9, p. 109, 2007.

[80] F. Benini, "A chiral cascade via backreacting D7-branes with flux," Journal of High Energy Physics, vol. 2008, no. 10, p. 051, 2008.

[81] F. Bigazzi, A. L. Cotrone, and A. Paredes, "Klebanov-Witten theory with massive dynamical flavors," Journal of High Energy Physics, vol. 2008, no. 9, p. 048, 2008.

[82] F. Bigazzi, A. L. Cotrone, A. Paredes, and A. V. Ramallo, "The Klebanov-Strassler model with massive dynamical flavors," Journal of High Energy Physics, vol. 2009, no. 3, p. 153, 2009.

[83] B. A. Burrington, J. T. Liu, L. A. P. Zayas, and D. Vaman, "Holographic duals of flavored $\mathrm{N}=1$ super Yang-Mills: beyond the probe approximation," Journal of High Energy Physics, vol. 2005, no. 2, p. 022, 2005.

[84] J. Evslin and S. Kuperstein, "Trivializing and orbifolding the conifold's base," Journal of High Energy Physics, vol. 2007, no. 4 , p. 001, 2007.

[85] D. Arean, D. Crooks, and A. V. Ramallo, "Supersymmetric probes on the conifold," Journal of High Energy Physics, vol. 2004, no. 11, p. 035, 2004. 